\title{
Sialic acid catabolism by $N$-acetylneuraminate pyruvate lyase is essential for muscle function
}

\author{
Xiao-Yan Wen,, Maja Tarailo-Graovac, ${ }^{3,4,5,6}$ Koroboshka Brand-Arzamendi,, ${ }^{1,2}$ Anke Willems, ${ }^{7}$ \\ Bojana Rakic, ${ }^{3}$ Karin Huijben, ${ }^{8}$ Afitz Da Silva, ${ }^{9}$ Xuefang Pan, ${ }^{9}$ Suzan El-Rass, ${ }^{1,2}$ \\ Robin Ng, ${ }^{1,2}$ Katheryn Selby, ${ }^{3}$ Anju Mary Philip, ${ }^{1,2}$ Junghwa Yun, ${ }^{1,2}$ X. Cynthia Ye, ${ }^{3,4}$ Colin J. Ross, ${ }^{3}$ \\ Anna M. Lehman, ${ }^{3}$ Fokje Zijlstra, ${ }^{8}$ A. Abu Bakar, ${ }^{7}$ Britt Drögemöller, ${ }^{10}$ Jacqueline Moreland, ${ }^{6}$ \\ Wyeth W. Wasserman, ${ }^{3,4}$ Hilary Vallance, ${ }^{3}$ Monique van Scherpenzeel, ${ }^{7,8}$ Farhad Karbassi, ${ }^{1,2}$ \\ Martin Hoskings, ${ }^{11}$ Udo Engelke, ${ }^{8}$ Arjan de Brouwer, ${ }^{12}$ Ron A. Wevers, ${ }^{8}$ Alexey V. Pshezhetsky,, 13 \\ Clara D.M. van Karnebeek, ${ }^{4,11,14}$ and Dirk J. Lefeber,8
}

'Zebrafish Centre for Advanced Drug Discovery and Keenan Research Centre for Biomedical science, Li Ka Shing Knowledge Institute, St. Michael's Hospital, Toronto, Ontario, Canada. 'Department of Medicine, Physiology, Laboratory Medicine and Pathobiology and Institute of Medical Science, Faculty of Medicine, University of Toronto, Ontario, Canada. ${ }^{3}$ Department of Medical Genetics and Department of Pathology and Laboratory Sciences, BC Children's Hospital Research Institute, University of British Columbia, Vancouver, Canada. ${ }^{4}$ Centre for Molecular Medicine and Therapeutics, Vancouver, British Columbia, Canada. ${ }^{5}$ Institute of Physiology and Biochemistry, Faculty of Biology, The University of Belgrade, Belgrade, Serbia. ${ }^{6}$ Departments of Biochemistry, Molecular Biology, and Medical Genetics, Cumming School of Medicine, Alberta Children's Hospital Research Institute, University of Calgary, Calgary, Canada. 'Department of Neurology, Donders Institute for Brain, Cognition, and Behavior, Radboud University Medical Center, Nijmegen, Netherlands. ${ }^{8}$ Translational Metabolic Laboratory, Department of Laboratory Medicine, Radboud University Medical Center, Nijmegen, Netherlands. ${ }^{9}$ SainteJustine University Hospital Research Center, University of Montreal, Montreal, Quebec, Canada. ${ }^{10} \mathrm{Faculty}$ of Pharmaceutical Sciences, University of British Columbia, Vancouver Canada. "Department of Pediatrics, BC Children's Hospital Research Institute, University of British Columbia, Vancouver Canada. ${ }^{2}$ Department of Human Genetics, Donders Institute for Brain, Cognition and Behaviour, Radboud University Medical Center, Nijmegen, Netherlands. ${ }^{13}$ Department of Anatomy and Cell Biology, McGill University, Montreal, Quebec, Canada. ${ }^{14}$ Departments of Pediatrics and Clinical Genetics, Emma Children's Hospital, Amsterdam University Medical Centres, University of Amsterdam, Amsterdam, The Netherlands.

Authorship note: XYW, AVP, CDMVK, and DJL contributed equally to this work. MTG, KBA, AW, and BR contributed equally to this work.

Conflict of interest: The authors have declared that no conflict of interest exists.

License: Copyright 2018, American Society for Clinical Investigation.

Submitted: May 24, 2018

Accepted: November 14, 2018

Published: December 20, 2018

Reference information:

JCI Insight. 2018;3(24):e122373.

https://doi.org/10.1172/jci.

insight.122373.
Sialic acids are important components of glycoproteins and glycolipids essential for cellular communication, infection, and metastasis. The importance of sialic acid biosynthesis in human physiology is well illustrated by the severe metabolic disorders in this pathway. However, the biological role of sialic acid catabolism in humans remains unclear. Here, we present evidence that sialic acid catabolism is important for heart and skeletal muscle function and development in humans and zebrafish. In two siblings, presenting with sialuria, exercise intolerance/muscle wasting, and cardiac symptoms in the brother, compound heterozygous mutations [chr1:182775324C>T (c.187C>T; p.Arg63Cys) and chr1:182772897A>G (c.133A>G; p.Asn45Asp)] were found in the $\mathrm{N}$-acetylneuraminate pyruvate lyase gene (NPL). In vitro, NPL activity and sialic acid catabolism were affected, with a cell-type-specific reduction of $\mathrm{N}$-acetyl mannosamine (ManNAc). A knockdown of NPL in zebrafish resulted in severe skeletal myopathy and cardiac edema, mimicking the human phenotype. The phenotype was rescued by expression of wild-type human NPL but not by the $p$.Arg63Cys or $p$.Asn45Asp mutants. Importantly, the myopathy phenotype in zebrafish embryos was rescued by treatment with the catabolic products of NPL: $\mathbf{N}$-acetyl glucosamine (GIcNAc) and ManNAc; the latter also rescuing the cardiac phenotype. In conclusion, we provide the first report to our knowledge of a human defect in sialic acid catabolism, which implicates an important role of the sialic acid catabolic pathway in mammalian muscle physiology, and suggests opportunities for monosaccharide replacement therapy in human patients. 


\section{Introduction}

Sialic acids, a group of structurally diverse, negatively charged, 9-carbon-chain sugar compounds are found in humans, animals, and bacteria, most commonly as terminal sugars in oligosaccharides. Sialic acids are important for a variety of biological functions including cell-cell interactions, bacterial and viral infections, and tumor metastasis (1).

Biosynthesis of endogenous sialic acid occurs in the cytosol, starting from UDP- $N$-acetyl glucosamine (UDP-GlcNAc). Epimerization and phosphorylation of UDP-GlcNAc by the bifunctional enzyme UDP- $N$-acetylglucosamine 2-epimerase/ $N$-acetylmannosamine kinase (GNE) yields ManNAc-6-phosphate, which is converted to $N$-acetylneuraminic acid (Neu5Ac) by $N$-acetylneuraminic acid synthase (NANS) and $N$-acetylneuraminic acid phosphatase (NANP), respectively. Subsequently, Neu5Ac is activated in the nucleus by $N$-acylneuraminate cytidylyltransferase (CMAS) to form cytidine-5-monophosphoneuraminic acid (CMP-Neu5Ac) (2). CMP-Neu5Ac is transported to the Golgi apparatus by SLC35A1 (3) for sialylation of glycoproteins and gangliosides catalyzed by specific sialyltransferases (4). Within the cytoplasm, CMP-Neu5Ac binds to GNE providing a negative feedback regulation of de novo Neu5Ac synthesis. Free Neu5Ac is also generated by the lysosomal salvage pathway, where sialic acids are released from sialoglycoconjugates by neuraminidases 1,3 , and 4 (5, 6). Neu5Ac is then transported to the cytosol by sialin, encoded by $S L C 17 A 5$, for synthesis of CMPNeu5Ac. Although the majority of research on the biological function of sialic acids has focused on the biosynthetic pathway, enzymes for Neu5Ac catabolism have been identified in humans and animals. Neuraminic acid pyruvate-lyase (NPL), also known as sialic acid aldolase (7), can act on Neu5Ac to form $\mathrm{N}$-acetylmannosamine (ManNAc) and pyruvate. ManNAc can be converted to $N$-acetylglucosamine (GlcNAc) by $N$-acetylglucosamine 2-epimerase (RENBP) and subsequently phosphorylated to GlcNAc-6-phosphate for entry into the hexosamine biosynthesis pathway. How these 3 pathways, i.e., biosynthesis, lysosomal salvage, and catabolism, are regulated and how each of them contributes to the levels of Neu5Ac and its metabolites remains unclear.

Important lessons on the biological role of Neu5Ac arose from genetic disorders (8). Two diseases caused by defects in CMP-Neu5Ac biosynthesis are known: GNE mutations result in an adult-onset myopathy (MIM 605820), whereas patients with NANS deficiency (MIM 610442) show intellectual disability and skeletal dysplasia (9). Both defects are thought to be caused by reduced production of CMP-Neu5Ac. Drastically increased Neu5Ac in urine, sialic aciduria, has been associated with 2 genetic conditions. Frenchtype sialuria (MIM 269921) due to defective feedback inhibition of GNE by CMP-Neu5Ac presents with a relatively mild lysosomal storage-like disorder. A defect in the lysosomal salvage pathway due to recessive mutations in SLC17A5 can result in a severe multisystem lysosomal storage disorder (MIM 269920) or a milder cognitive disease (Salla disease, MIM 604369). Although the clinical phenotypes of these disorders are considerably different, progressive muscle involvement is present in GNE myopathy and as hypotonia in SLC17A5 deficiency. Heart involvement has been reported sporadically in both syndromes.

Here, we describe a human genetic defect in sialic acid catabolism due to biallelic mutations in NPL, resulting in sialic aciduria and a clinical phenotype of progressive cardiac myopathy and mild skeletal myopathy. We demonstrate dysfunction of the mutant NPL enzyme and a block in sialic acid catabolism and document a phenotype in a zebrafish NPL model that mimics the human clinical presentation. Furthermore, we show that the muscle phenotype in the $n p l$ zebrafish model could be rescued by supplementation with the monosaccharide products of the NPL reaction. Combined, our data indicate the importance of the Neu5Ac catabolic pathway for human muscle physiology.

\section{Results}

A cause of sialic aciduria with heart and skeletal muscle dysfunction. A 22-year-old male proband (P1.1) was a second child of healthy nonconsanguineous Filipino parents. During the pregnancy, fetal arrhythmia (third degree heart block) with hydrops was diagnosed, and delivery was induced at 33 weeks gestation. At birth, the left ventricle was enlarged with normal output. Sensorineural hearing loss was also noticed. Subsequently he developed progressive dilated cardiomyopathy with left ventricular ejection fraction of only $20 \%$, which was complicated by cardiac arrest. At the age of 16 years, he demonstrated mild joint hypermobility, retrognathia, and thin musculature with a mild proximal myopathy in upper and lower limbs (MRC grade 4/5). During metabolic analysis a marked increase in urinary sialic acid was identified. The patient's older female sibling (P1.2), who refused clinical examination, has a history of exercise intolerance and a mild 
muscle weakness. She also has marked sialic aciduria. The levels of urinary Neu5Ac were in the range of patients with Salla disease (Figure 1A); however, no accumulation of Neu5Ac in fibroblasts could be detected (Figure 1B). In addition, no clinical resemblance was found with Salla disease or French-type sialuria. Metabolic investigations showed normal sialylation of transferrin and apolipoprotein CIII (data not shown).

In searching for the genetic cause, trio (proband-mother-father) whole exome sequencing (WES) and prioritization of genetic variants were performed as described in the Methods section (10). Rare, nonsynonymous genetic variants were identified in 26 genes following different modes of Mendelian inheritance (Supplemental Table 1; supplemental material available online with this article; https://doi.org/10.1172/ jci.insight.122373DS1). Of these, only the 2 missense variants of the NPL gene, involved in sialic acid catabolism, could be related to the sialic aciduria in our patients: chr1:182775324C > T (c.187C > T; p.Arg63Cys), NM_030769, with reported ExAC 4/121286 and gnomAD 7/277158 occurrence; and chr1:182772897A>G (c.133A>G; p.Asn45Asp), NM_030769, with reported ExAC 112/121088 and gnomAD 242/277152 occurrence; Figure 1, C-E). Both variants were predicted to be damaging by SIFT (11) and Polyphen2 (12), with CADD scores of 34 and 21.4 (13), respectively. The same variants were found by Sanger sequencing in the affected sister with trans parental inheritance (Figure 1C). Aside from NPL, variants in the other 25 genes were further excluded as candidate genes for sialic aciduria on the basis of segregation analysis, pathogenicity prediction score, mode of inheritance, and/or known phenotype and gene functions (Supplemental Table 1). No additional patients with causative mutations in NPL could be identified in the exome data of a multicenter cohort of approximately 2,000 patients with neuromuscular disease.

p.Arg63Cys and p.Asn45Asp mutations affect enzymatic activity and stability of NPL. To study the effect of mutations, wild-type (WT) or mutant NPL variants containing a C-terminal DYK tag were expressed in HEK293T cells. To verify equal transfection efficiencies, the cells were cotransfected with the pCMV-HGSNAT-GFP plasmid (14). Forty-eight hours after transfection, NPL activity in the cell homogenates was measured against Neu5Ac, $N$-glycolylneuraminic acid (Neu5Gc), or 2-keto-3deoxy-D-glycero-D-galacto-nononic acid (KDN), as described by Brunetti et al. (15). Cells transfected with the WT NPL plasmid showed an approximately 16-fold increase in Neu5Ac lyase activity as compared with untransfected cells. The activity in the cells expressing the p.Asn45Asp and p.Arg63Cys NPL mutants was significantly lower, approximately $30 \%$ and approximately $6 \%$ of the WT, respectively (Figure 2A). In addition, the p.Asn45Asp NPL mutant had 3.2-fold increased $K_{m}$ and 2.6-fold decreased $V_{\max }$ values as compared with the WT enzyme (Figure 2B), suggesting that the mutation decreased the enzyme affinity for Neu5Ac approximately 8-fold, most probably affecting its function inside the cell. The activity of the WT NPL against Neu5Gc was 5-fold lower than that against Neu5Ac (Supplemental Figure 1) and below the detection limit for the mutants. Neither WT enzyme nor mutants had any activity against KDN (data not shown).

The protein expression levels of the WT NPL and its mutants were tested by Western blot using antiDYK tag antibodies that detected a single $36-\mathrm{kDa}$ protein band with expected size. We found (Figure 2C) that the expression level of the p.Asn45Asp NPL mutant, although significantly lower then that of the WT NPL, was reduced only to approximately $60 \% \pm 13 \%$, suggesting that the mutation mainly affects the enzymatic activity. The expression level of the p.Arg63Cys NPL mutant was reduced to approximately $25 \% \pm$ $4 \%$ of the WT NPL, suggesting that the mutation interferes with expression and/or stability of the enzyme. To test this directly, we studied the stability of the WT NPL and its mutants by nonradioactive pulse-chase in which we blocked de novo protein synthesis in the transfected cells and measured intensity of the NPL protein band at 2, 4, 6, 18, and 24 hours by Western blot. Our results (Figure 2D) show that the cellular half-life of p.Asn45Asp and p.Arg63Cys NPL mutants (19.6 and 7.3 hours, respectively) is significantly lower than that of the WT enzyme (161 hours).

p.Arg63Cys and p.Asn45Asp mutations alter Neu5Ac metabolism in patient tissues. A potential dysfunction of NPL was studied by incubating homogenates of P1.1's fibroblasts and red blood cells (RBCs) with Neu5Ac. Quantitative mass spectrometric analysis showed a time-dependent decrease of Neu5Ac in control RBCs. In contrast, RBCs of P1.1 from 2 independent sampling dates did not show any catabolism of Neu5Ac (Figure 3A). No activity was detected in human control fibroblasts, which is in agreement with the absence of sialic acid accumulation in the patient's cultured fibroblasts. The analysis of free Neu5Ac in the patient's RBCs revealed an accumulation of approximately 50- to 100-fold as compared with control cells (262.5 pmol/mg protein, reference range 2.5-5.8), consistent with the catabolic dysfunction of NPL in RBCs (Supplemental Table 2). 
A

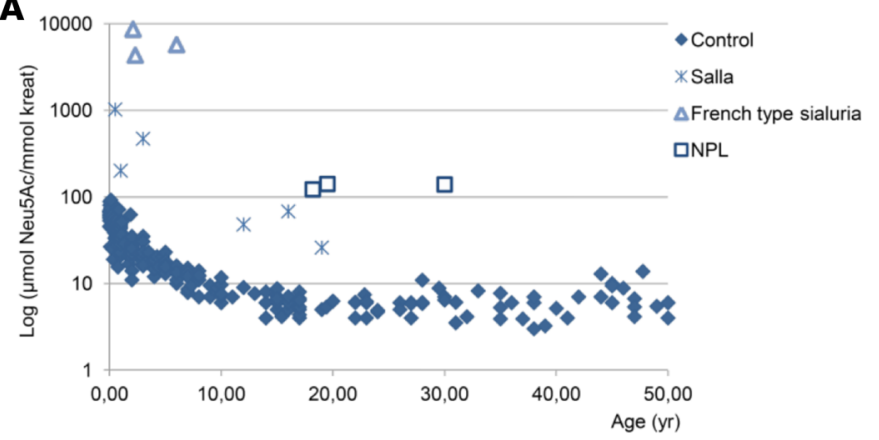

B

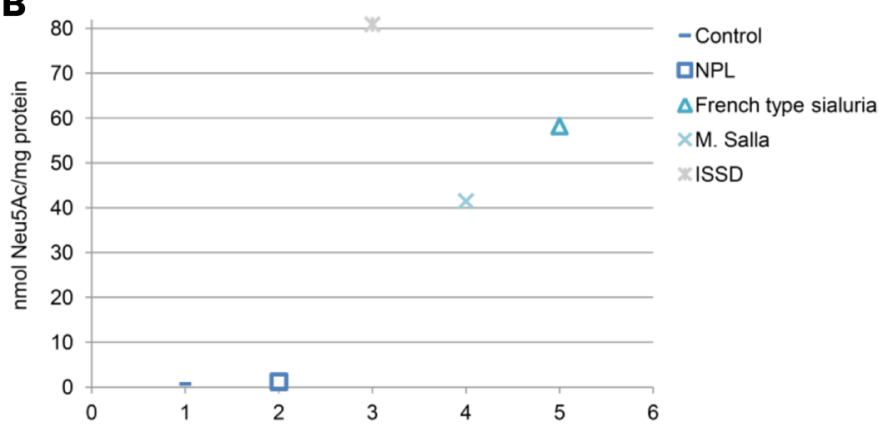

C
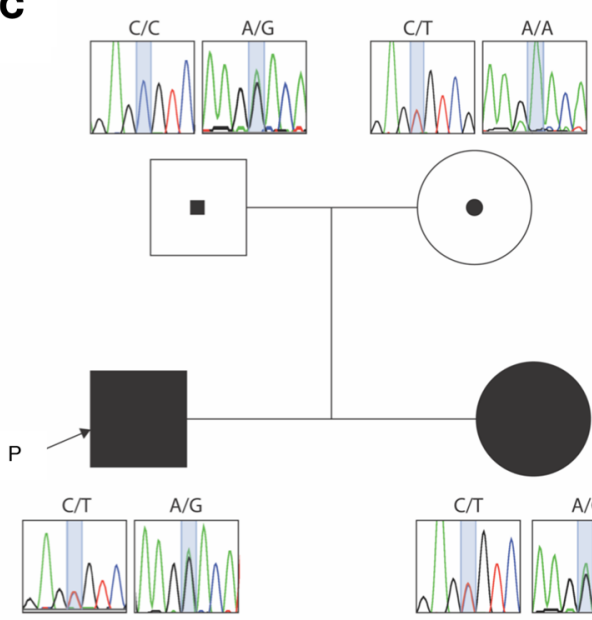

D
Human
Zebrafish
Mouse
Dog
Chicken
Xenopus
Alpaca
Mutant 1
Mutant 2

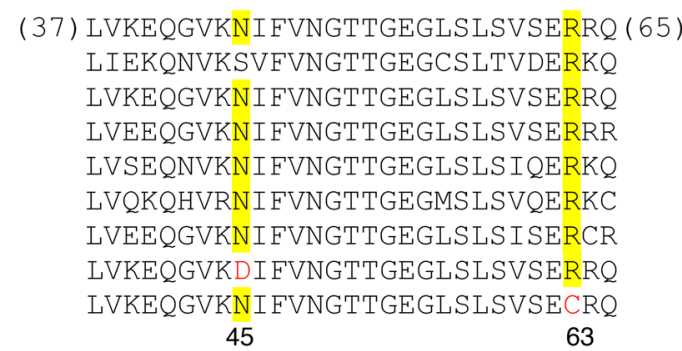

45

E

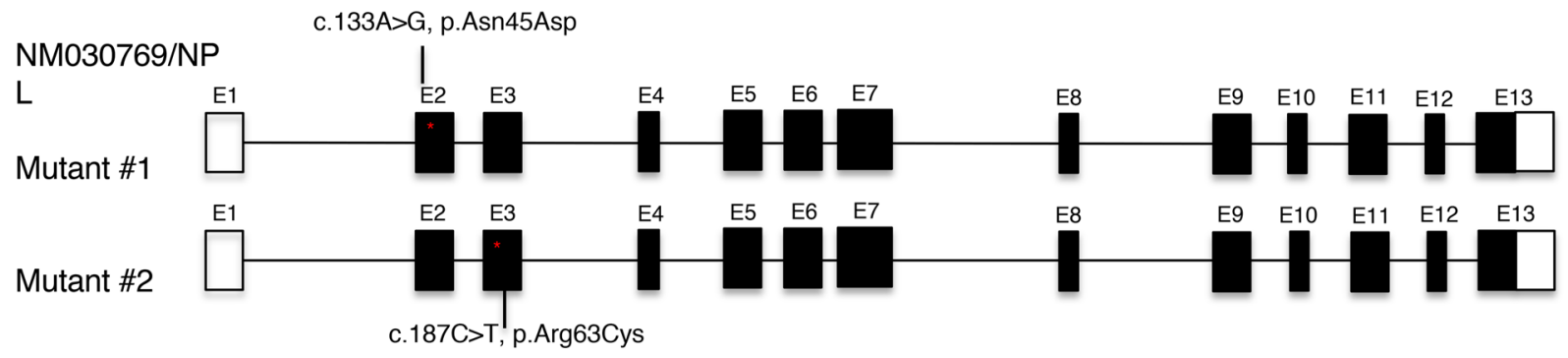

Figure 1. NPL deficiency and identification of NPL biallelic missense varants in NPL. (A) Sialic aciduria was determined by quantification of the levels of urinary Neu5Ac excretion and compared with known causes of sialic aciduria. For NPL patient 1, two samples were analyzed at around age 20 years. Salla refers to both ISSD at a very young age and Salla disease. (B) As part of the diagnostics of sialic aciduria, quantification of Neu5Ac levels in fibroblasts was performed, showing normal levels in contrast to known causes (all $n=1$ ). (C) Whole exome sequencing, covering $37.8 \times$ for the subject, $30.4 \times$ for the mother, and $27.5 \times$ for the father, to identify NPL missense varants. (D) Comparison of the amino acid sequences of NPL in human (Q9BXD5.1), zebrafish (CAP19481.1), mouse (NP_083025.1), dog (XP_005622466.1), chicken (NP_001026731.1, Xenopus (NP_001011207.1), and alpaca (XP_015101171.1), as well as 2 mutants. Conserved asparagine (N) and arginine (R) are highlighted. Mutations are shown in red. (E) Biallelic NPL variants c.133A>C (p.Asn45Asp) and c.187C >T (p.Arg63Cys) (reference transcript NM_030769) are mapped on NPL exon 2 and 3, respectively.

Metabolomics analysis (16) confirmed free Neu5Ac accumulation in urine and plasma. To further investigate the effect of NPL deficiency on the downstream Neu5Ac metabolites, we first studied a possible reduction of ManNAc levels in body fluids. Levels of ManNAc were low in urine and plasma of both patient P1.1 and controls (data not shown). We then analyzed the intracellular levels of ManNAc, ManNAc-6-phosphate, and Neu5Ac via a mass spectrometric method adapted from Buescher et al. (17). A clear reduction of ManNAc and ManNAc-6-phosphate levels was observed in patient RBCs, but not in fibroblasts (Figure 3, B-G), indicating that the levels of Neu5Ac, ManNAc, and ManNAc-6-phosphate are strongly dependent on NPL only in specific cell types. 
A
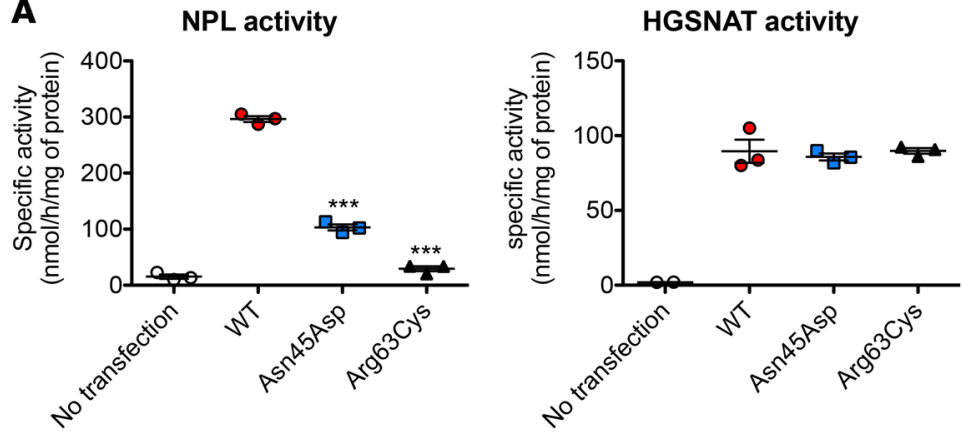

B

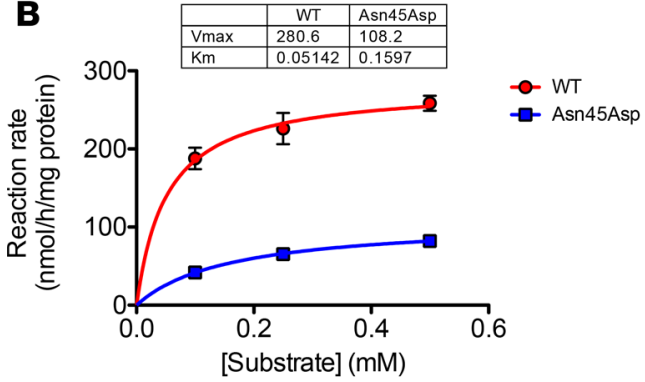

C

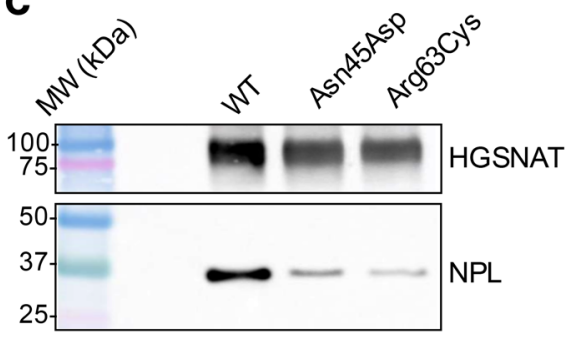

HGSNAT activity
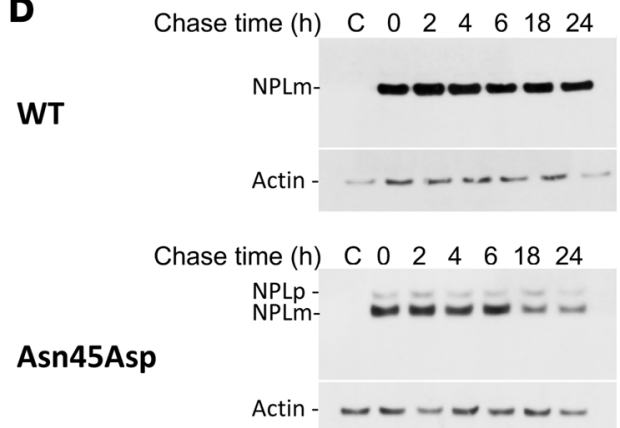

Chase time (h) $\quad$ C $\quad 0 \quad 2 \quad 2 \quad 4 \quad 6 \quad 1824$
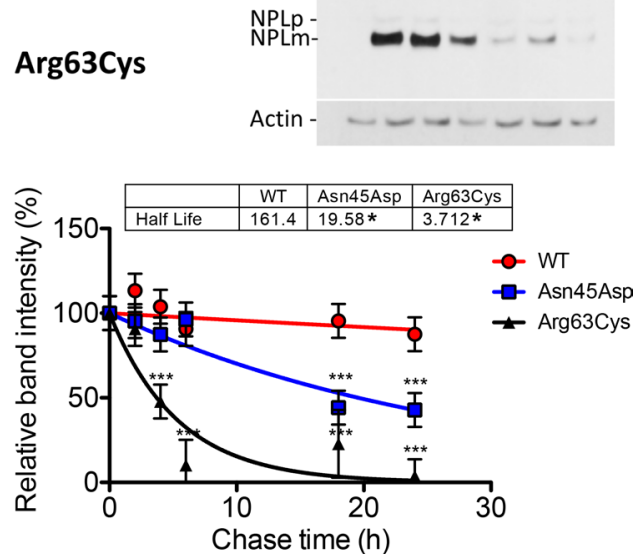

Figure 2. p.Asn45Asp and p.Arg63Cys mutations affect enzymatic activity and expression and/or stability of the NPL protein. (A) Cultured HEK293T cells cotransfected with plasmids encoding cDNA of the wild-type (WT) human NPL or its p.Arg63Cys and p.Asn45Asp mutants containing a C-termina DYK tag and pCMV-HCSNAT-GFP plasmid encoding human acetyl-CoA: $\alpha$-glucosaminide $N$-acetyltransferase (HCSNAT). Forty-eight hours after transfection, the cells were harvested and the enzymatic NPL and HCSNAT activities were measured in cell homogenates. (B) The reaction rate of Neu5Ac hydrolysis by the WT NPL and the p.Asn45Asp NPL mutant were measured at different initial concentrations of the substrate to calculate the $K_{m}$ and $V_{\text {max }}$ values for the WT and mutant enzyme. (C) The protein levels of WT NPL and its mutants were measured by Western blot using antibodies against the DYK tag. Panels show representative images of 3 independent experiments. The bar graph shows intensities of the DYK cross-reactive protein bands (means \pm SD of the values from 3 independent experiments) measured with Imagel software and normalized to the intensities of the control HGSNAT protein band stained with anti-HGSNAT antibody. (D) Stability of WT NPL protein and its mutants was measured by nonradioactive pulsechase experiment. HEK293T cells expressing WT or mutant NPL were treated with $7 \mu$ M cycloheximide to inhibit de novo protein synthesis, chased 2 , $4,6,18$, and 24 hours, and analyzed by Western blot to measure intensity of the NPL protein band (DYK tag). Panels show representative images of 3 independent experiments; 20 and $60 \mu \mathrm{g}$ of protein was loaded per well for the cells transfected with the WT and mutant plasmids, respectively. Bands representing mature NPL (NPLm) and its precursor (NPLp) are marked. The graph shows intensities of the DYK cross-reactive protein bands (means \pm SD of the values from 3 independent experiments) measured with Image software. Table shows half-life of WT and mutant NPL protein (hours) calculated using 1-phase decay nonlinear regression. ${ }^{*} P<0.05,{ }^{* *} P<0.01$, ${ }^{* *} P<0.001$ versus WT by 1 - and 2 -way ANOVA.

Altogether, our data clearly demonstrate the deleterious impact of the compound heterozygous variants in NPL as detected in both patients, causing impairment of Neu5Ac catabolism resulting in its accumulation and free sialic aciduria.

$N p l$ knockdown in zebrafish mimics the human phenotype and can be rescued with human NPL but not its p.Arg63Cys and p.Asn45Asp mutants. Given that the expression pattern of $n p l$ in zebrafish is unknown, we performed in situ hybridization on zebrafish embryos at different developmental stages. We found that $n p l$ is already expressed in 1-cell-stage embryos, indicating maternal contribution (Figure 4A). In addition, $n p l$ is strongly expressed in somites and embryonic muscle in 18-somite-stage embryos (Figure 4A). At 24 hours postfertilization (hpf), $n p l$ presents a more restricted expression pattern, with higher expression in the head (Supplemental Figure 2). Expression of $\mathrm{npl}$ in the intestine is also evident at $48 \mathrm{hpf}$, 


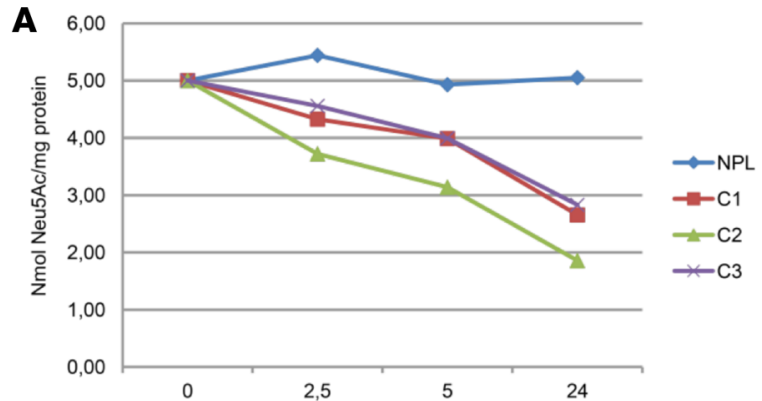

B $\bullet \mathrm{RBC}$
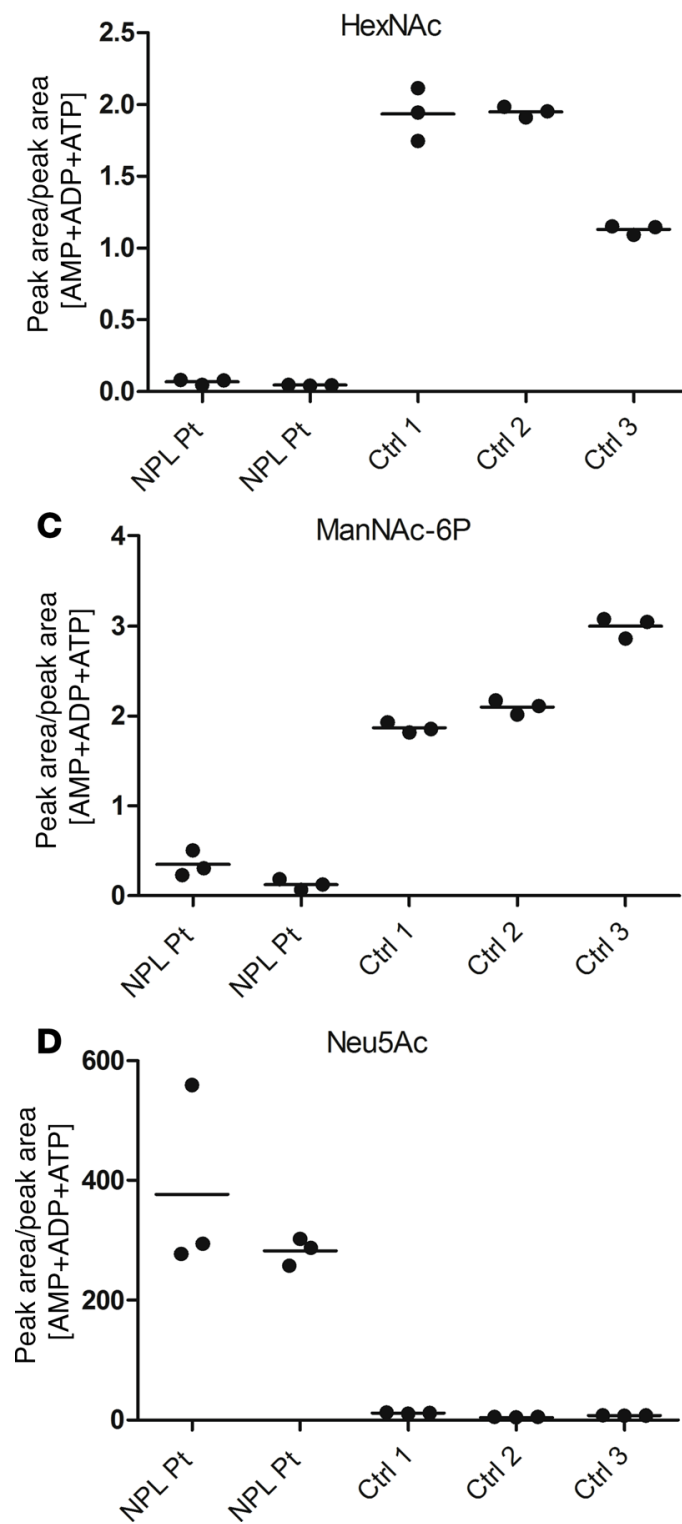
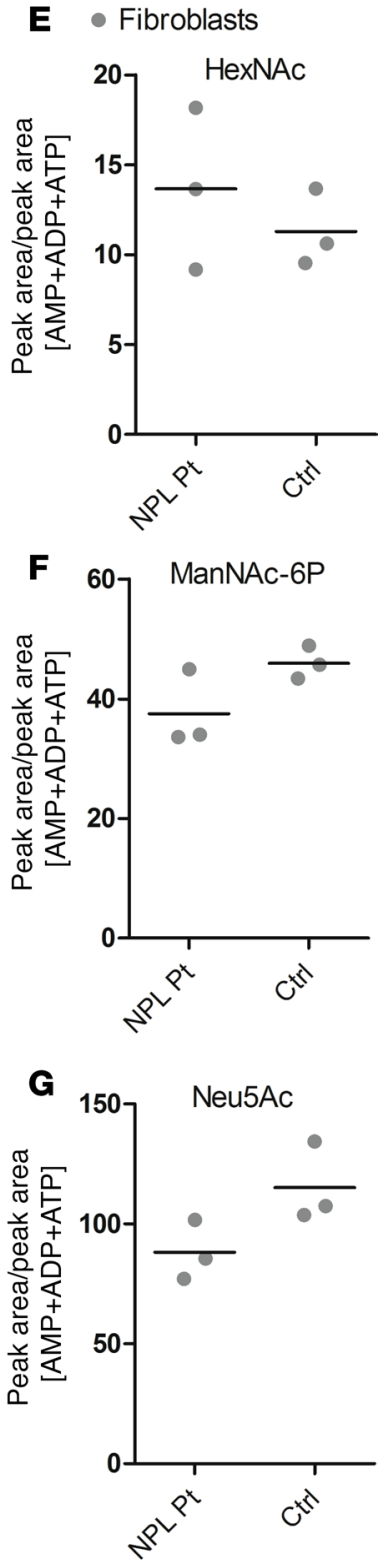

Figure 3. Neu5Ac catabolism and NPL metabolites in patient cells. (A) Neu5Ac catabolism was analyzed in patient RBCs by incubation of control or NPL patient 1-derived RBCs with 500 $\mu \mathrm{M}$ Neu5Ac. Neu5Ac levels after different time points were quantified by mass spectrometry. Metabolites of the NPL reaction were analyzed in RBCs (B-D) and fibroblasts (E-C). Dot plots represent peak areas of HexNAc (B and $\mathbf{E}$ ), ManNAc-6-phosphate (C and F), and Neu5Ac ( $\mathbf{D}$ and $\mathbf{G}$ ), normalized against the sum of AMP, ADP, and ATP. Separate dots represent technical replicates. Separate samples represent independent drawings of blood (patient RBCs) or different donors (ctrl RBCs).

as well as at 3 and 4 days postfertilization (dpf). In addition, $n p l$ is detected in facial musculature and the limb bud at 3 and $4 \mathrm{dpf}$ (Supplemental Figure 2). Hybridization with a sense probe rendered a negligible signal (Figure 4A), confirming the specificity of the $n p l$ anti-sense probe. For comparison, we also performed expression analysis of the NPL gene in cDNA pools of human fetal and adult tissues. Highest expression was observed in spleen, while other tissues, including heart and skeletal muscle, showed relatively similar expression levels (data not shown). Overall, the expression pattern of $n p l$ is consistent with a possible function in muscle development. 
To determine whether deficiency of npl affects muscle development and structure, we knocked down $n p l$ gene expression in zebrafish embryos by injecting a morpholino oligonucleotide targeting the ATG codon ( $n p l$-atg MO) to block translation. $n p l$ morphants, but not embryos injected with a standard control morpholino (Cont MO), were found to have mild pericardial edema, curved tail, and somite disorganization (Figure 4B). Western blotting (Figure 4C) and NPL enzymatic assay (Supplemental Figure 3) confirmed efficient npl knockdown in morphants. To assess somite morphology and muscle fiber organization, we performed confocal imaging on 48-hpf morphants and Cont MO-injected embryos stained with phalloidin, which reveals actin filaments. This analysis demonstrated abnormal somite morphology with disarrayed muscle fibers in npl morphants (Figure 4D). In situ hybridization for myod, a skeletal muscle marker (18), confirmed abnormal somite morphology in npl morphants at $24 \mathrm{hpf}$ and $48 \mathrm{hpf}$ (Supplemental Figure 4). In addition, myod staining revealed absence of ocular and facial muscles in morphants at $48 \mathrm{hpf}$.

To further validate the functional rescue of the muscle phenotype, we studied the locomotion behavior of 2-dpf zebrafish larvae by a touch response. As shown in Figure 5, A and B, npl-atg MO-injected zebrafish larvae demonstrate decreased motor behavior in response to touch, in terms of total distance moved and maximum velocity swam. Coinjection of WT mRNA in the $n p l$ morphants or supply of ManNAc in embryo water significantly improves swim behavior of the $n p l$-atg MO-injected larvae (Figure 5, A and B).

A recent study demonstrated that GNE myopathy is associated with muscle oxidative stress in humans and mice (19). To study the role of oxidative stress in npl-associated myopathy, we analyzed reactive oxygen species (ROS) levels in zebrafish trunk muscles in vivo (Figure 5C). The npl-atg morphants showed a 2-fold increase in ROS levels in the trunk muscles compared with the embryos injected with Cont MO. In contrast, coinjection of $n p l$-atg MO with WT human NPL mRNA significantly lowered ROS levels. Furthermore, incubation of $n p l$-atg morphants with ManNAc also rescued and lowered ROS to control levels (Figure 5D).

To further confirm the involvement of npl in muscle development, we knocked down npl using a splicing morpholino targeting the exon 4/intron 4 boundary (npl-sp MO). As expected, morphants displayed abnormal somite morphology along with disarrayed muscle fibers, and pericardial edema (Supplemental Figure 5). Reverse transcription PCR (RT-PCR) on Cont MO produced a 260-bp band corresponding to the length of properly spliced exons 3, 4, and 5. In contrast, an enlarged product consistent with retention of intron 4 was detected in $n p l$-sp MO morphants (Supplemental Figure 5, A and C).

Pericardial edema is often a sign of cardiac dysfunction. Therefore, we analyzed heart morphology in $n p l$ morphants. Epifluorescence images of embryos carrying the cmlc2:GFP transgene, which labels cardiomyocytes, revealed abnormal looping of the heart tube in the $n p l$ morphants (Figure 4E).

To exclude potential off-target effects of the $n p l$-atg MO, we evaluated whether coinjection with human $N P L$ mRNA rescues the phenotype in morphants. In both NPL mRNA-injected and control morphants we assessed somite morphology and muscle fiber arrangement. As expected, injection of the npl-atg $\mathrm{MO}$, but not the Cont $\mathrm{MO}$, resulted in a drastic decrease in the percentage of embryos with normal somite morphology. Sixty-six percent of embryos injected with the npl-atg MO (Figure 6, C and D), and less then 1\% of embryos injected with Cont MO, had abnormal somite morphology (Figure 6, A and B, and Figure 7). In contrast, coinjection of $n p l$-atg MO with WT human NPL mRNA significantly increased the percentage of embryos with normal muscle morphology (77\%; Figure 6, E and F, and Figure 7), suggesting that the injected human NPL mRNA rescued the $n p l$-atg MO phenotype in zebrafish.

To further demonstrate that NPL p.Asn45Asp and p.Arg63Cys are disease-causing mutations, we synthesized mutant NPL minigenes carrying single or double (p.Asn45Asp plus p.Arg63Cys) mutations and generated mutant mRNA by in vitro transcription, and performed mRNA rescue experiments (Figure 6 , $\mathrm{A}-\mathrm{T}$, and Figure 7). Injection of double-mutant NPL mRNA did not rescue the $n p l$-atg MO-induced phenotype, as $72 \%$ of embryos showed myopathy (Figure 6, I and J, and Figure 7). Injection of p.Asn 45 Asp or p.Arg63Cys mutant mRNA also yielded at least $42 \%$ of embryos with myopathy in both cases (Figure 6, M, N, Q, and R, and Figure 7), providing additional indication that p.Asn45Asp and p.Arg63Cys are disease-causing mutations.

NPL-myopathy is improved by monosaccharide replacement in zebrafish, offering options for therapeutic intervention. In view of the suggested catabolic function of NPL, we hypothesized that the downstream monosaccharide products of the NPL reaction, ManNAc and/or GlcNAc, could rescue the npl morphant phenotype. Embryos injected with Cont $\mathrm{MO}$ or $n p l$-atg MO were treated with ManNAc, GlcNAc Neu5Ac, and control monosaccharides (D-galactose, D-mannose, or D-xylose) followed by analysis of somite and heart morphology and 


\section{$A$
0
0
0
0
0
.1
.$\frac{1}{c}$
$\frac{1}{2}$
$\frac{1}{1}$}
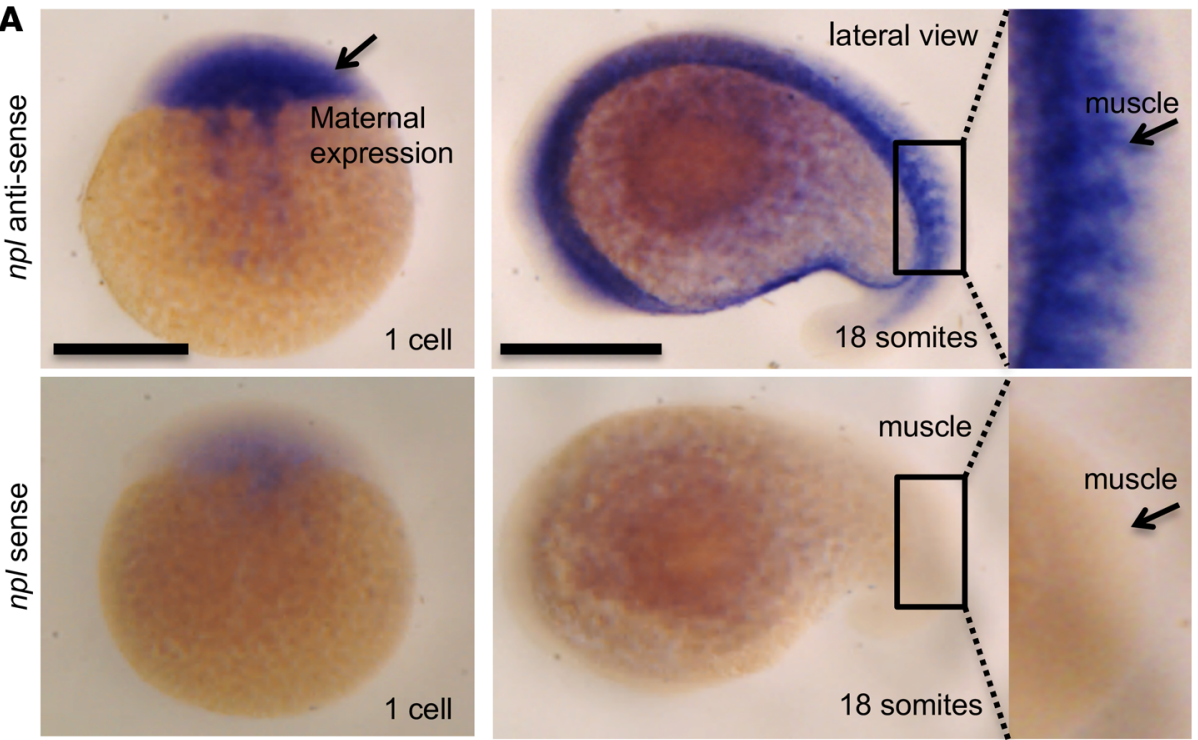

B
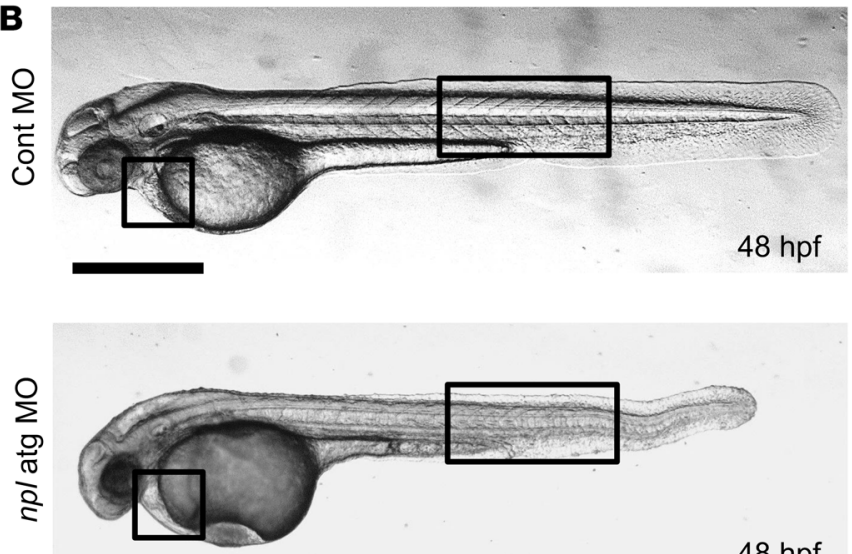

D

Cont $\mathrm{MO}$

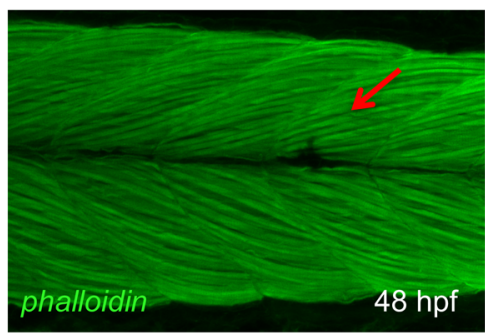

$n p / \operatorname{atg} \mathrm{MO}$

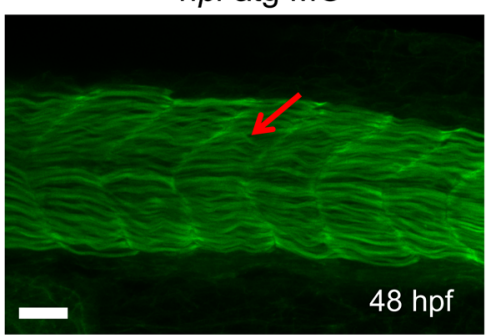

E

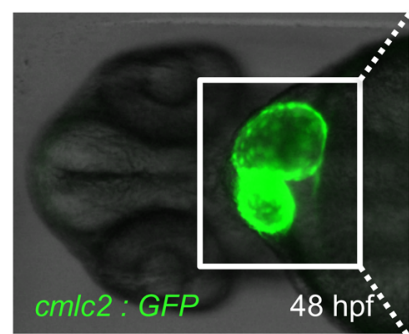

\section{$n p /$ atg $\mathrm{MO}$}

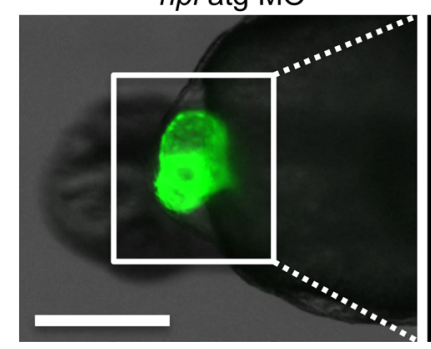

$48 \mathrm{hpf}$
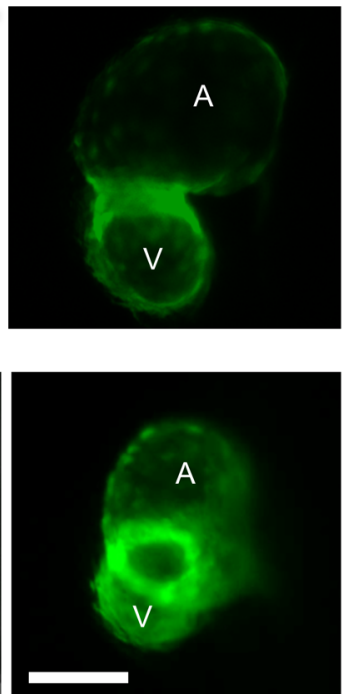

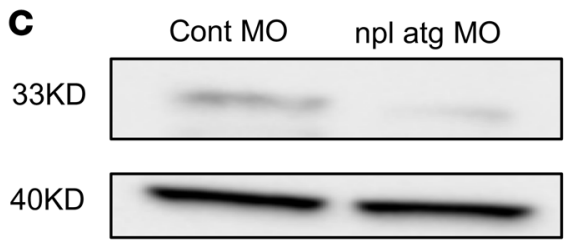

NPL protein

beta-actin protein dorsal view

18 somites
Figure 4. $n p l$ is essential for muscle development in zebrafish. (A) Wholemount in situ hybridization of $\mathrm{np}$ / on WT embryos using antisense and control sense probes. In situ hybridization showing $\mathrm{npl}$ expression (arrow) in 1-cellstage, indicating maternal expression, and 18 -somite embryos. $\mathrm{npl}$ is broadly expressed in embryos at the 18-somite stage. Arrows in lateral view point to somites in closeup. The experiment was repeated 3 times. Scale bars: $200 \mu \mathrm{m}$ (left) and $250 \mu \mathrm{m}$ (middle). (B) Lateral views of zebrafish embryos at 48 hours postfertilization (hpf) that were injected with control (Cont) and atg morpholino (MO) oligonucleotides against $\mathrm{npl}$ (npl-atg). Severe somite disorganization and mild pericardial edema (boxes) were observed. Scale bar: $750 \mu \mathrm{m}$. (C) Western blot of NPL in Cont MO- and npl-atg MO-injected embryos; $\beta$-actin was used as loading control. (D) Confocal images of muscle fibers in somites (arrows) immunostained for phalloidin in Cont MO and npl atg MO. Scale bar: $50 \mu \mathrm{m}$. (E) Epifluorescence images of the heart in cmlc2:GFP transgenics at $48 \mathrm{hpf}$ injected with Cont $\mathrm{MO}$ and $\mathrm{npl}$-atg MO. Boxes delimit close-up views of hearts. Scale bars: $100 \mu \mathrm{m}$ (left) and $25 \mu \mathrm{m}$ (right). 

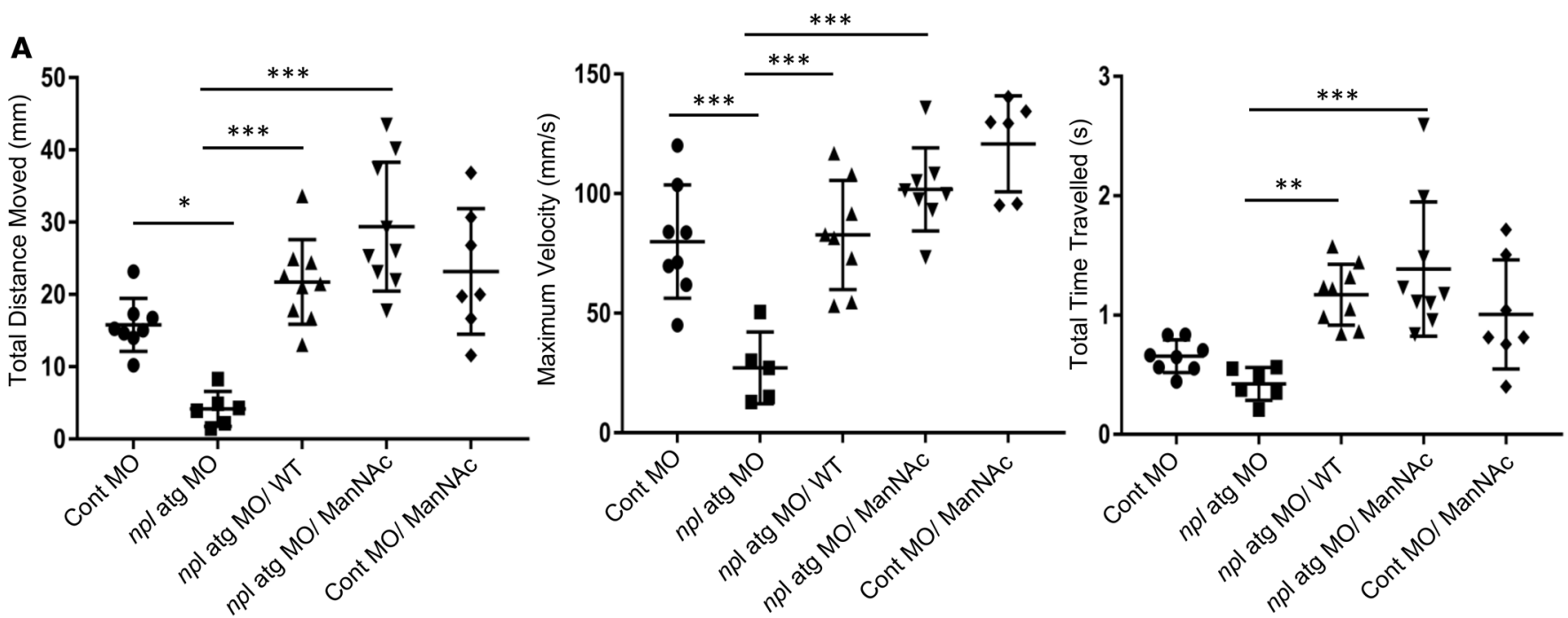

B Cont $\mathrm{MO}$

$n p l$ atg $\mathrm{MO}$

$n p /$ atg MO/ WT

$n p l$ atg $\mathrm{MO} / \mathrm{ManNAc}$

Cont $\mathrm{MO} / \mathrm{ManNAC}$

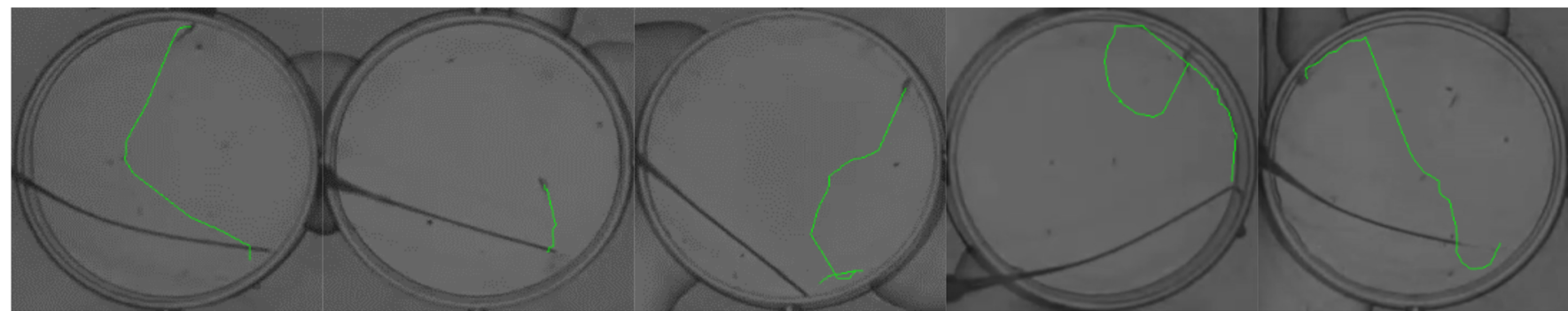

C

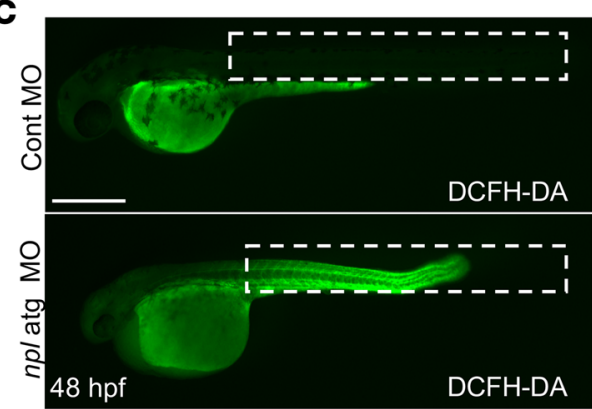

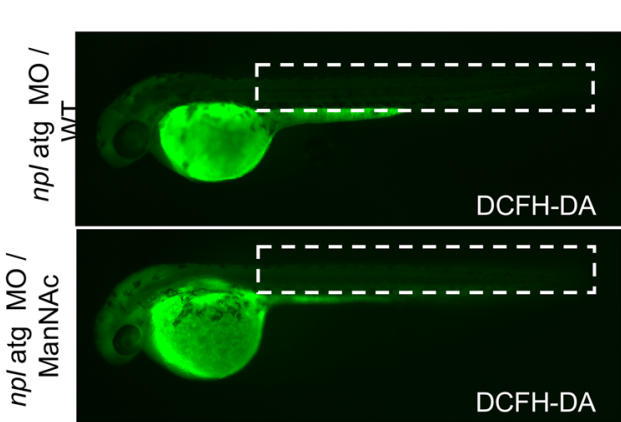

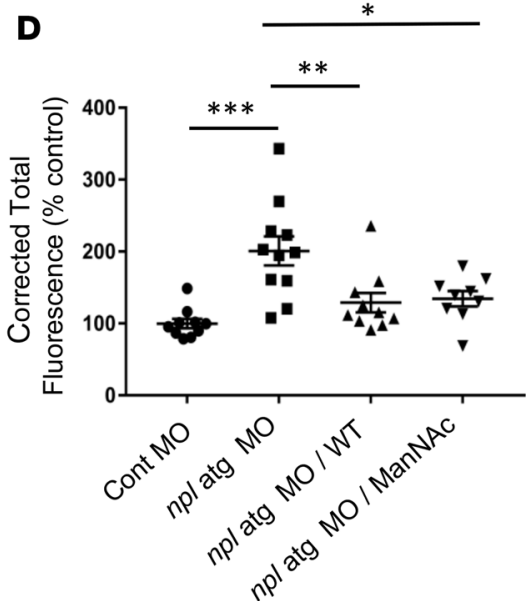

Figure 5. Rescue of zebrafish npl deficiency in morphant embryos by NPL mRNA or ManNAc. (A) Behavior rescue in a touch response assay. Coinjection of WT mRNA or incubation in $800 \mu \mathrm{M}$ ManNAc solution significantly improved swim behavior of $n p l$-atg morphant larvae in terms of total distance moved, maximum velocity, and total time travelled. Error bars represent the standard deviation of 6-9 larvae. Significance was determined using a linear mixed-effects model with $\mathrm{npl}$-atg MO injection conditions (fixed effect) and individual larvae (random effect), with Bonferroni's post hoc test. (B) Representative paths swam in response to touch for groups of Cont MO, npl-atg MO, npl-atg MO/NPL WT mRNA, npl-atg MO/ ManNAc, and Cont MO/ManNAc zebrafish larvae. Videos are attached as supplemental materials. (C) Myopathy in $\mathrm{npl} \mathrm{morphants} \mathrm{is} \mathrm{associated} \mathrm{with}$ increased ROS levels in skeletal muscle. Increased fluorescence in $\mathrm{npl}$ mutants revealed by a ROS indicator is rescued by WT NPL mRNA injections or ManNAc treatment. (D) Quantification of fluorescence relative to Cont $\mathrm{MO}$ embryos in embryos injected with $\mathrm{npl}$-atg $\mathrm{MO}, \mathrm{npl}$-atg $\mathrm{MO}$ coinjected with WT human (NPL mRNA WT), or $n p l$-atg MO incubated with ManNAc. Bars represent the mean \pm SE. $n=10-11$ embryos per treatment. Statistical significance was determined using 1-way ANOVA with Bonferroni's post hoc test assuming equal variance using GraphPad Prism software version 7. ${ }^{*} P<0.05,{ }^{* *} P<0.01,{ }^{* *} P<0.001$. 
A

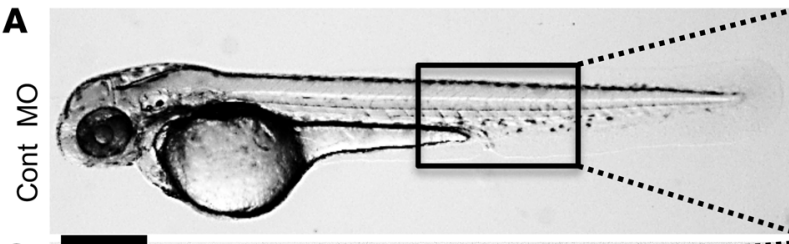

C

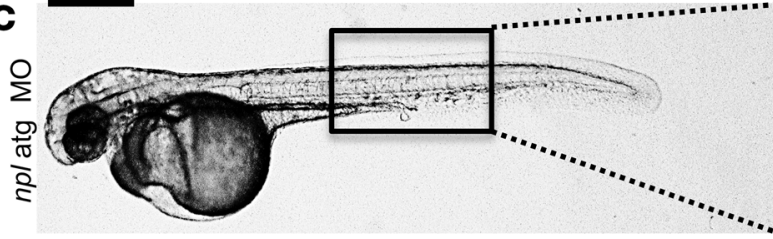

$\mathbf{E}$
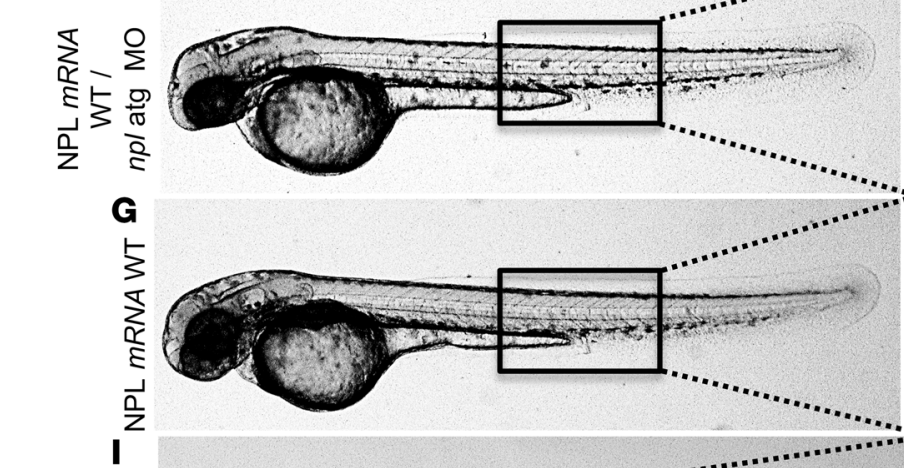

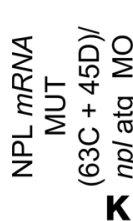

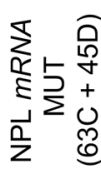
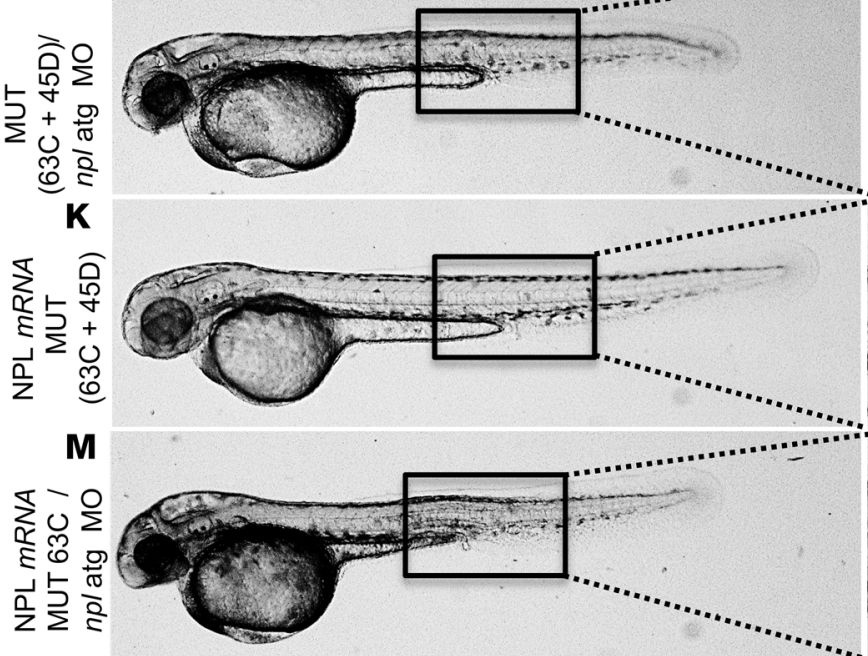

M

0

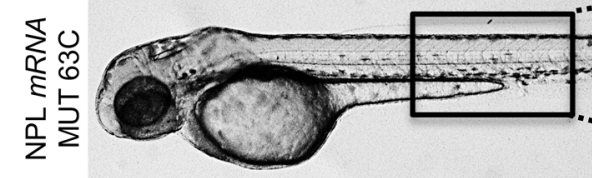

Q

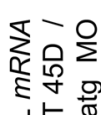

로 $\frac{1}{2}$

\section{$\mathbf{S}$}

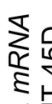

온$$
\text { ... }
$$
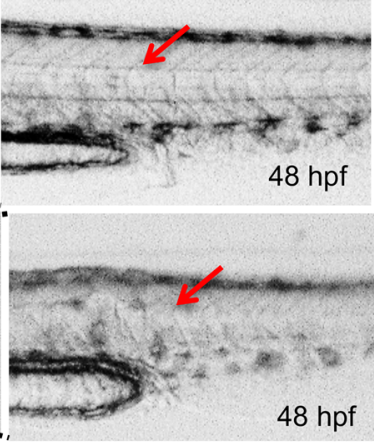

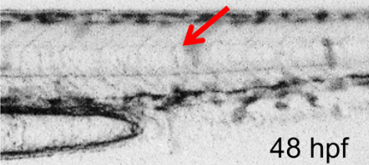

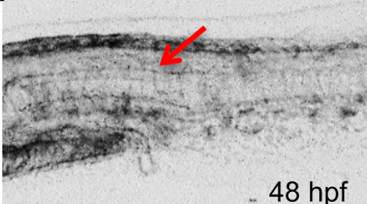

48 hpf

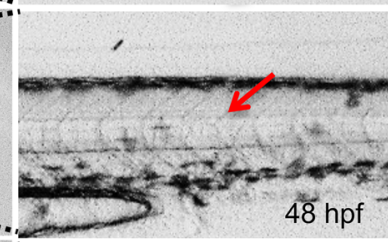

$\mathbf{J}$

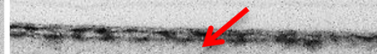

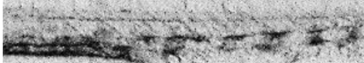
48 hpf$$
\therefore
$$

एकic -

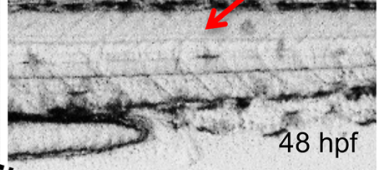

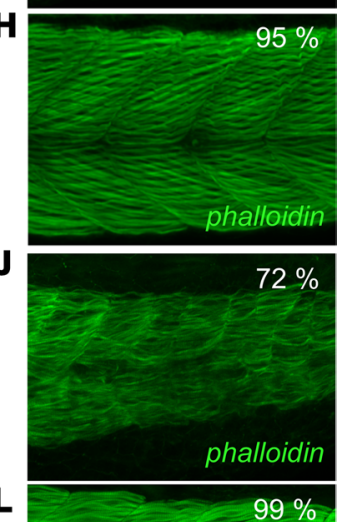

$\mathbf{L}$

$\mathbf{L}$
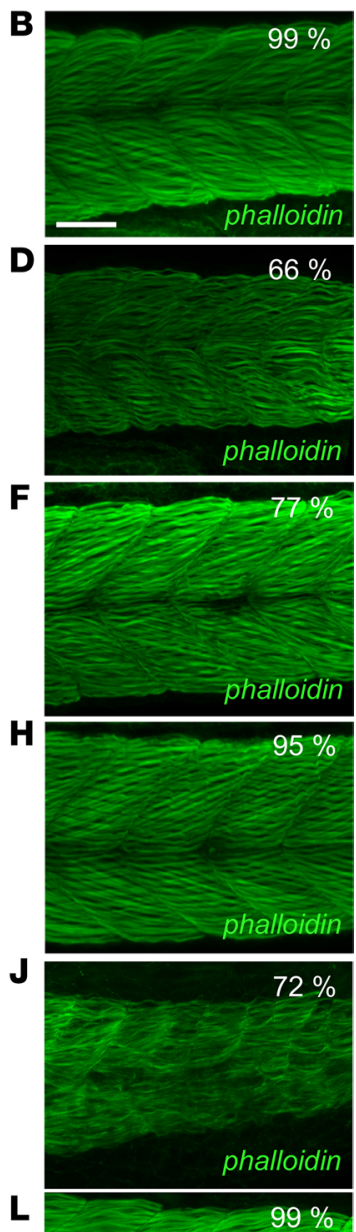

$\mathbf{F}$
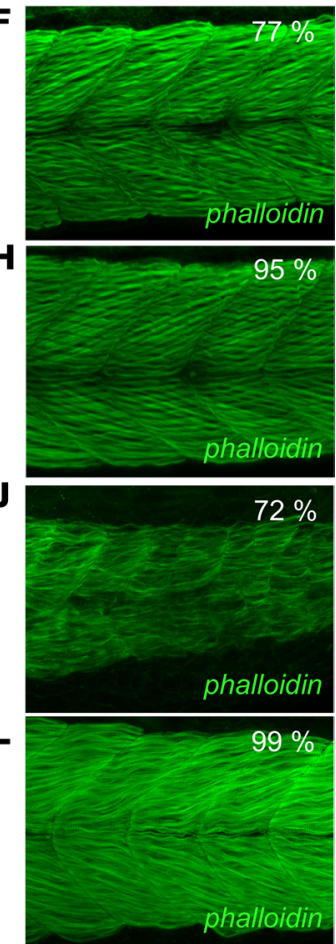

$\mathbf{N}$
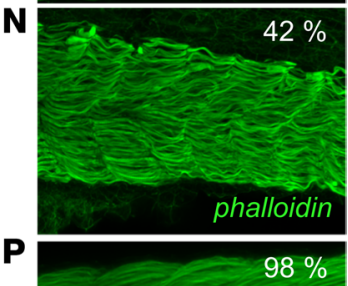

$98 \%$

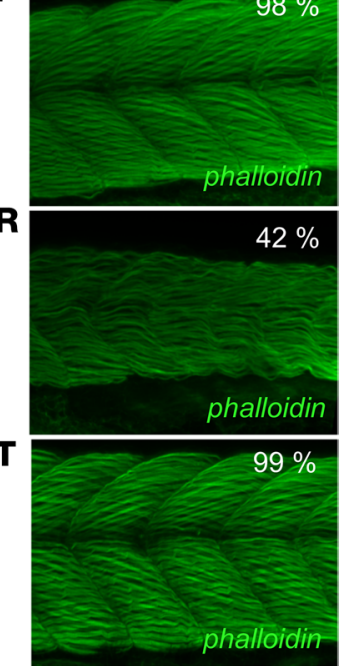


Figure 6. $n$ pl morphants are rescued by injection of human WT but not the mutant NPL mRNA. Lateral views and closeups (boxed areas) of embryos with or without skeletal myopathy after injection of (A and $\mathbf{C}$ ) Cont $\mathrm{MO}$ or $\mathrm{npl}$-atg $\mathrm{MO}$ alone, or (E) npl-atg $\mathrm{MO}$ in combination with WT human or (G) (NPL mRNA WT) alone. (I) Embryos coinjected with human NPL mRNA carrying a double mutation (63C $+45 \mathrm{D})$ and $n p l$-atg MO; or (K) NPL mRNA MUT (63C + 45D) human NPL mRNA; or (M) human NPL mRNA with a single mutation for 63C (MUT 63C) and npl-atg MO; or (Q) 45D (MUT 45D) and $\mathrm{npl}$-atg MO; or ( $\mathbf{O}$ and $\mathbf{S}$ ) a single mutation (63C or 45D). Closeups (boxed areas) show somites (arrows). Scale bar: $750 \mu \mathrm{m}$. (B, D, F, $\mathbf{H}, \mathbf{J}, \mathbf{L}, \mathbf{N}, \mathbf{P}, \mathbf{R}, \mathbf{T}$ ) Confocal images of muscle fibers in somites (arrows) immunostained for phalloidin on 48 -hpf embryos. Scale bar: $50 \mu \mathrm{m}$. The experiment was repeated at least 3 times.

muscle fiber arrangement. Incubation with ManNAc and GlcNAc resulted in a significant increase in the percentage of embryos with normal somite morphology in npl-atg morphants (Figure 8, A, B, and G). ManNAc was the most efficient, with $88 \%$ of $n p l$-atg MO embryos rescued (Figure 8 , A and G), followed by GlcNAc with $72 \%$ rescue (Figure $8, \mathrm{~B}$ and $\mathrm{G}$ ). Neu5Ac (sialic acid) could also rescue the muscle phenotype to a lesser extent (46\%, Figure 8, C and G), possibly attributable to the residual activity of NPL, which allows increased production of ManNAc upon feeding with Neu5Ac. Control monosaccharides did not provide any rescue of the muscle phenotype (Figure 8, D-G). Furthermore, confocal imaging of somites immunostained with phalloidin, and cmlc2:GFP transgenic embryos showed that incubation with ManNAc, and GlcNAc, and to a lesser extent with Neu5Ac, improved muscle fiber arrangement. Feeding with ManNAc but not with GlcNAc or Neu5Ac significantly improved heart looping studied by both stereo- and confocal microscopy (Figure 9A-D). Furthermore, incubation of $n p l$-atg morphants with ManNAc demonstrated a rescued phenotype of locomotion behavior deficit (Figure 5, A and B) and restored relatively normal ROS levels, comparable to controls (Figure 5, C and D). ManNAc also reestablished normal somite morphology and muscle fiber array in $n p l$-sp MO morphants (Supplemental Figure 5D).

\section{Discussion}

Here, we report the first evidence to our knowledge for a physiological role of sialic acid catabolism (Figure 10) and its downstream metabolites ManNAc and GlcNAc in mammalian muscle physiology. Compound heterozygous missense variants were identified in NPL in human siblings, and shown to cause its dysfunction in vitro and in vivo. A biological role of sialic acid catabolism independent from glycosylation of proteins and lipids was demonstrated by the rescue of the muscle and heart phenotype of $n p l$ zebrafish morphants via administering ManNAc.

In mammals, activity of $N$-acetylneuraminate lyase has been detected in different tissues including liver, kidney, spleen, brain, lungs, and erythrocytes, but its biological function remained unclear. Our in vitro and in vivo experimental data strongly support the conclusion that the biallelic NPL variants, p.Arg63Cys and p.Asn45Asp, are pathogenic and responsible for the biochemical and clinical phenotype of the patients. First, both NPL proteins carrying either p.Arg63Cys or p.Asn45Asp substitution expressed in cultured human HEK293T cells had significantly reduced enzymatic NPL activity against Neu5Ac ( $\sim 6 \%$ or $\sim 30 \%$ of the WT, respectively). NPL activity in the patient's RBCs was below the detectable level and the Neu5Ac levels were approximately 50- to 100-fold increased as compared with healthy controls, confirming that the mutations disrupt in vivo NPL activity and sialic acid catabolism. On the other hand, the low-to-undetectable NPL activity in control human fibroblasts suggests variable tissue NPL expression and tissue-specific demands for sialic acid catabolism. Second, knockdown of the $n p l$ gene in developing zebrafish embryos showed skeletal myopathy and cardiac edema, which could be rescued by human WT NPL mRNA but not by mutants. The cardiac phenotype that we observed in $n p l$ morphants is not surprising, as many genes involved in human and mouse cardiovascular function and development were previously knocked down in zebrafish resulting in cardiac edema and/or looping defect phenotypes (20). Importantly, such phenotypes can be rescued by pharmacological intervention with existing drugs (21).

Three signature parts of the NPL enzyme are sequences located in amino acid regions 42-63, 79-97, and 138-155 (http://www.ebi.ac.uk/interpro/protein/Q9BXD5) and are presumably important for the enzymatic activity. The mutations p.Asn45Asp and p.Arg63Cys are both in the first signature region. Asparagine 45 is fairly conserved among different species (Figure 1D). The p.Asn45Asp mutation is very close to one of the active-site residues (p.Phe47) and so it may alter their positions, causing the reduced activity and affinity for the substrate. Our results showed that substitution of arginine 63 with cysteine significantly reduces the enzymatic activity (Figure 2A). Arginine 63 is highly conserved in NPL (Figure 1D) and located in an $\alpha$-helix in proximity to one of the dimer interface residues (p.Ser57) 


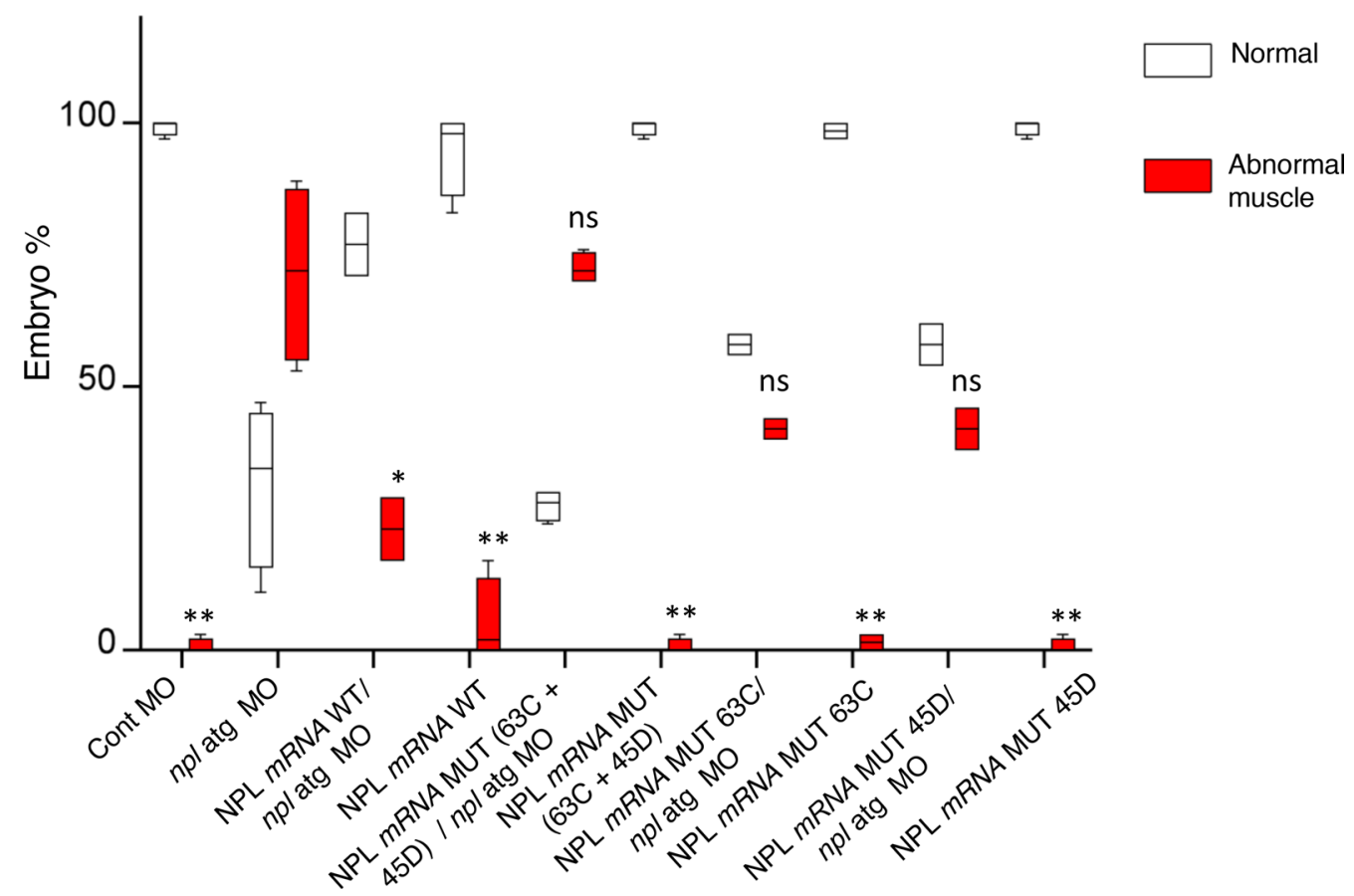

Figure 7. Percentage of $n p l$ morphants rescued by injection of human WT but not mutant NPL mRNA. Percentage of embryos with or without skeletal myopathy obtained after injection of Cont $\mathrm{MO}$ or $n \mathrm{~m} /$-atg $\mathrm{MO}$ alone, or npl-atg MO in combination with WT human (NPL mRNA WT), or mutant [NPL mRNA MUT (63C + 45D)] human NPL mRNA, or human NPL mRNA with a single mutation for 63C (MUT 63C), or 45D (MUT 45D). Bars represent the mean \pm SD of 4 independent injections; 10 groups were preselected for comparison. Statistical significance determined by 2-tailed Student's $t$ test with Bonferroni's correction with a factor of 10 using GraphPad Prism software version 7. ${ }^{*} P<0.05$, ${ }^{*} P<0.01$; ns, not significant. For simplicity, only comparisons to the $n p l$-atg MO abnormal muscle group are represented in the graph. Each bar represents between 50 and 135 embryos. The experiment was repeated 4 times.

(http://www.ebi.ac.uk/interpro/protein/Q9BXD5). Substitution of a positively charged amino acid (arginine) that has a long side chain, with the smaller neutral cysteine residue could destabilize the $\alpha$-helical structure (22). On the other hand, this mutation could potentially perturb the interaction between the subunits and hence the quaternary structure of the enzyme important for activity and stability.

In bacteria, NPL has been extensively studied and was thought to play an important role in controlling the intracellular concentration of sialic acid $(7,23,24)$ to prevent the buildup of its toxic levels and/or as a source for energy production. In humans, an additional role of NPL could be to control the levels of CMPNeu5Ac for protein sialylation, which is not present in most bacteria.

In the process of cell aging, NPL was proposed to eliminate the excess Neu5Ac generated. In addition, human NPL can catabolize exogenous Neu5Gc (25). This form of sialic acid is predominant in animals, but cannot be synthesized by humans due to the lack of CMP-Neu5Gc hydroxylase (CMAH). Large amounts of Neu5Gc are, however, consumed with dairy and meat products and are incorporated into glycoproteins that can cause an autoimmune response. Interestingly, both mutations in the reported patient completely abolish the enzymatic activity against Neu5Gc, suggesting that NPL deficiency could potentially result in its increased incorporation into protein glycans.

Increased Neu5Ac levels are associated with 2 other forms of free sialic aciduria with a considerably different clinical phenotype. Mutations in the lysosomal sialic acid transporter encoded by SLC17A5 result in lysosomal accumulation of free sialic acid and cause either a severe infantile sialic acid storage disease (ISSD) or a slowly progressing juvenile Salla disease. The main clinical symptoms in the patients are hypotonia, cerebellar ataxia, and intellectual disability; visceromegaly and coarse features are also present in the infantile cases. Cardiomegaly has also been reported (26). The loss of feedback control of GNE epimerase activity by CMP-NeuAc due to a mutation at the enzyme's allosteric site results in uncontrolled production of sialic acid and autosomal dominant Frenchtype sialuria. Only 7 patients so far have been reported in the literature (27). The disorder is perhaps 

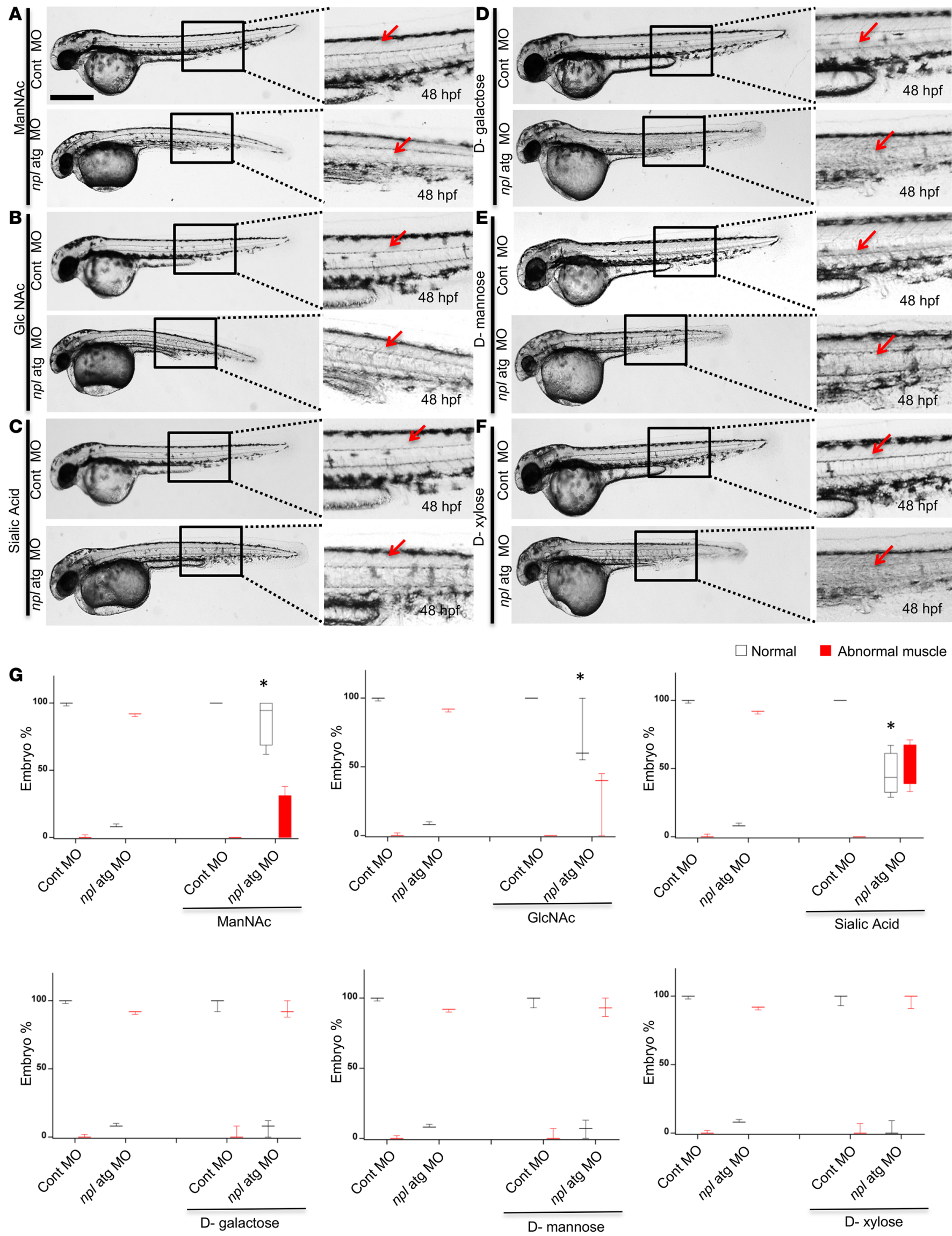
Figure 8. Myopathy in $\mathrm{npl}$ morphants is partially rescued by monosaccharide replacement. (A-F) Lateral views of embryos injected with Cont $\mathrm{MO}$ or $n p l-$ atg MO. Embryos were incubated with $800 \mu \mathrm{M}$ of the following monosaccharides: $N$-acetyl-D-mannosamine (ManNAc), $N$-acetyl-D-glucosamine (ClcNAc), sialic acid, D-galactose, D-mannose, or D-xylose, which were added to the water 2 hours after morpholino injection and incubated for 48 hours. Scale bar: $750 \mu \mathrm{m}$. Closeups of boxed areas show somites (arrows). (C) Percentage of embryos with or without skeletal myopathy obtained after injection with Cont $\mathrm{MO}$ or $\mathrm{npl}$-atg MO and treatment with ManNAc, GlcNAc, sialic acid, D-galactose, D-mannose, or D-xylose. Bars represent the mean \pm SD of 4 independent injections. Significance was determined using a 2-tailed $t$ test assuming equal variance data. ${ }^{*} P<0.05$. All comparisons were against $n p l$-atg $\mathrm{MO}$ normal muscle group. Between 25 and 180 embryos were analyzed per condition in 3 independent experiments.

underdiagnosed, as patients have relatively mild phenotype. The fact that French-type sialuria does not present with skeletal muscle involvement or cardiomyopathy with a much higher increase of sialic acid as compared with NPL deficiency is not supportive of the hypothesis that cytosolic accumulation of sialic acid itself leads to the NPL phenotype.

Our data suggest that the disease in NPL-deficient patients is triggered by changes in the cytosolic levels of downstream Neu5Ac metabolites, primarily ManNAc and GlcNAc. The skeletal myopathy in $n p l$-morphant zebrafish was rescued by ManNAc, the direct downstream metabolite of NPL, and to a lesser extent by G1cNAc and Neu5Ac. In contrast, rescue of the heart phenotype was only possible with ManNAc. It has been shown that ManNAc can be converted to GlcNAc-6-phosphate, likely via subsequent action of RENBP and GlcNAc kinase. This compound could be metabolized further to generate fructose-6-phosphate (Fru-6-phosphate) or to generate UDP-GlcNAc, the precursor for glycosylation reactions. In analogy to the role of bacterial NPL in energy production, Fru-6-phosphate could enter glycolysis for production of ATP. The lower efficiency of GlcNAc in rescuing the npl-knockdown phenotype, especially the heart phenotype, does not seem to match this hypothesis. However, the difference in the action of GlcNAc and ManNAc could be related to specifics of their uptake and transport. In addition, no other metabolic pathways are known to act directly on ManNAc. It is also noted that the best 2 rescuing monosaccharide compounds ManNAc and GlcNAc are uncharged, but Neu5Ac is negatively charged. Thus, Neu5Ac may enter embryos and cells less efficiently than the other sugars. Irrespective of the mechanism, our data imply a clear potential for the use of these monosaccharides as a novel treatment strategy.

Interestingly, ManNAc and Neu5Ac have also been proposed as treatment for GNE myopathy (28). Over 100 mutations have been reported in GNE, encoding UDP- $N$-acetylglucosamine-2-epimerase/ $N$-acetylmannosamine kinase, resulting in an adult-onset progressive myopathy with a characteristic pattern of affected muscles; although the hamstring and tibialis anterior muscles are affected severely by early adulthood, the quadriceps muscles are spared even at a late stage of the disorder (28). Cardiac involvement has been reported in GNE myopathy, although sporadically (29). Reduced protein sialylation has been reported in several studies. In the mouse model of the disease, muscle loss was prevented by treating animals with Neu5Ac or ManNAc. ManNAc was proposed to increase the synthesis of Neu5Ac via phosphorylation by a hexNAc kinase and subsequent action of NANS and NANP. This will increase CMP-Neu5Ac levels to improve protein sialylation. Since ManNAc also rescues the muscle myopathy caused by NPL deficiency in our zebrafish model, it is tempting to speculate that overlapping biochemical mechanisms may be involved in both diseases.

Skeletal muscles continually produce low levels of ROS due to their contractile activity and high oxygen consumption and metabolic rate. However, under normal conditions, multiple antioxidant systems help to keep ROS levels in check and maintain redox homeostasis (30). Recent studies show that dysregulated redox homeostasis has emerged as a key pathogenic condition in several muscle diseases like muscular dystrophy, GNE myopathy, and other early-onset myopathies $(19,30)$. Here we show that NPL myopathy is also associated with dysregulated ROS production. However, since ROS levels could be restored by ManNAc, we believe that enhanced ROS production in NPL myopathy is more likely caused by tissue responses to the primary pathology, in contrast with GNE myopathy where enhanced ROS is believed to be upstream of muscle atrophy.

Altogether, our data demonstrate an important biological role of NPL in cardiac and skeletal muscle function, and suggest that a reduced level of its downstream metabolite ManNAc is the cause of the cardiac and skeletal muscle phenotype. The results also suggest that supplementation with the commonly used dietary sugar GlcNAc could be explored as a potential therapeutic approach for treatment of NPL deficiency. More research is required to reveal the exact pathological mechanism of the myopathic phenotype, the intrafamilial variability in penetrance, and therapeutic strategies, which will also yield novel insights into understanding and treatment of other glycosylation-related myopathies. 
A
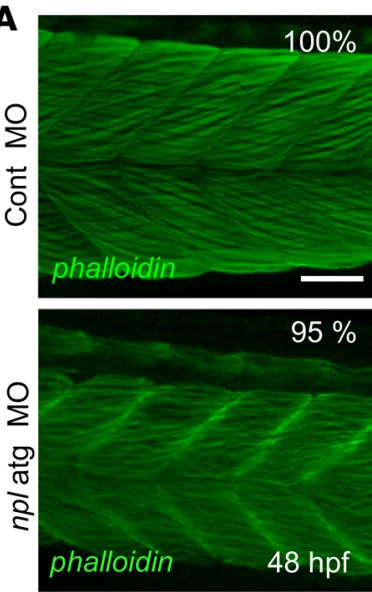

B

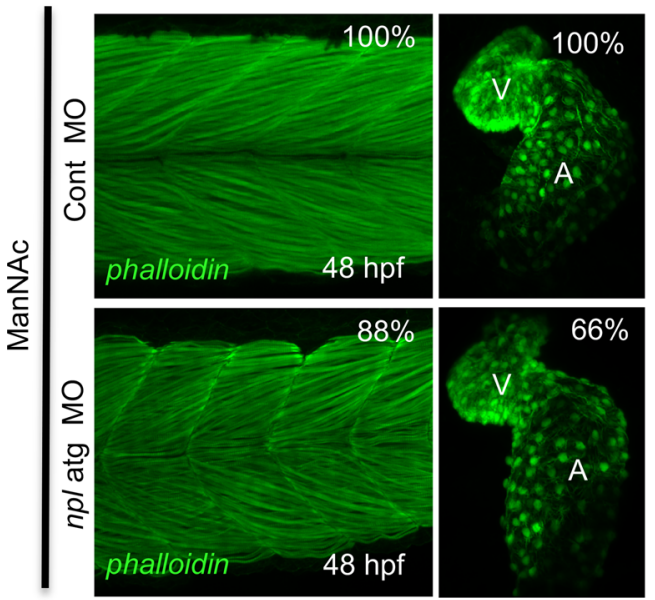

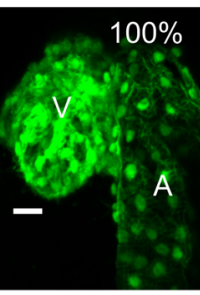

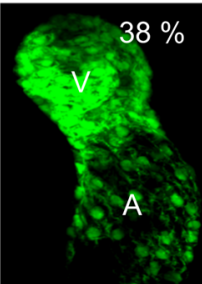

\section{D}

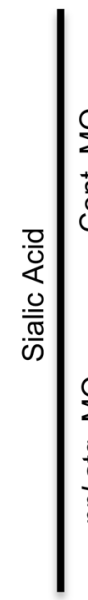

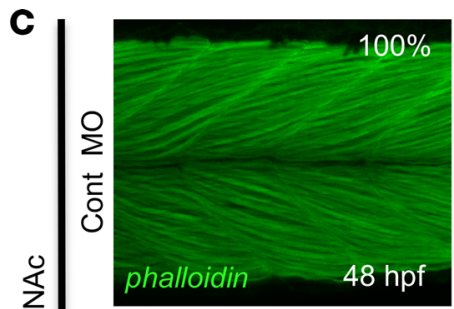
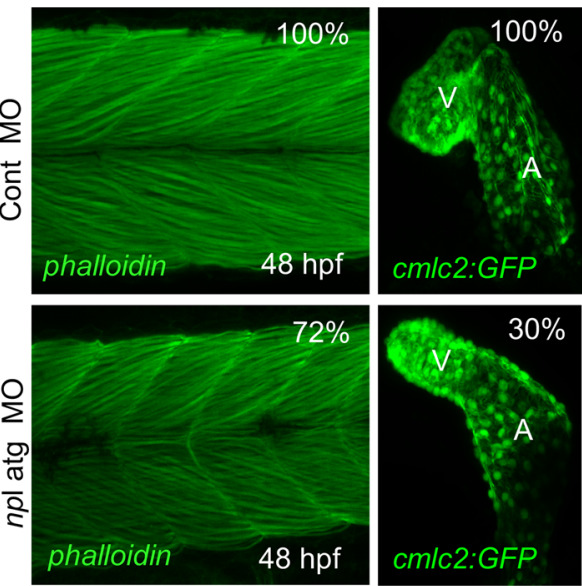

$\mathrm{cm} / \mathrm{c} 2: \mathrm{GFP}$
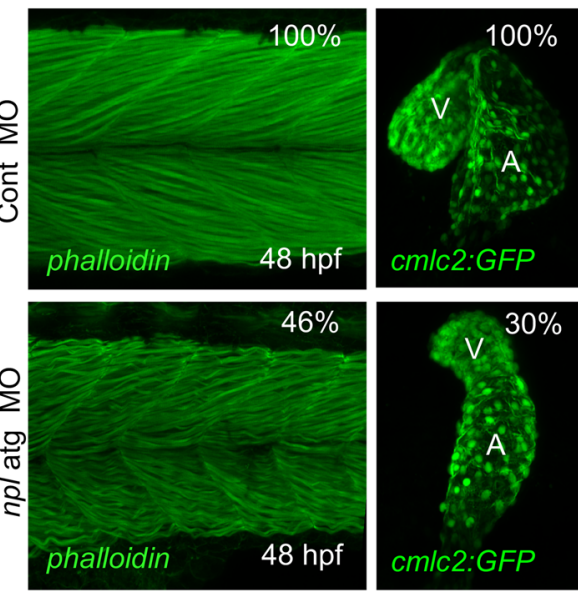

Figure 9. Monosaccharide replacement partially rescues skeletal but not cardiac myopathy in $n p l$ morphants. (A-D) Confocal images of muscle fibers in somites immunostained for phalloidin on 48-hpf embryos that were injected with Cont $\mathrm{MO}$ or $\mathrm{npl}$-atg MO. Embryos were incubated with $800 \mu \mathrm{M}$ of the following monosaccharides: ManNAc, GlcNAc, or sialic acid, which were added to the water 2 hours after morpholino injection and incubated for 48 hours. Left panels show epifluorescence images of the heart in cmlc2:GFP transgenics at $48 \mathrm{hpf}$. Between 3 and 5 embryos were analyzed per condition in 3 independent experiments. Scale bars: $50 \mu \mathrm{m}$ (left) and $25 \mu \mathrm{m}$ (right).

\section{Methods}

Human samples. Skin fibroblasts were obtained with informed consent from the patients (P1.1 and P1.2) and healthy controls and cultured at $37.0^{\circ} \mathrm{C}$ under $5.0 \% \mathrm{CO}_{2}$ in $\mathrm{E} 199$ culture medium, supplemented with $10 \%$ fetal calf serum and $1 \%$ penicillin/streptomycin. All cultures were negative for mycoplasma infection.

WES. The family was enrolled into the TIDEX gene discovery project (University of British Columbia IRB approval H12-00067), and provided written consent for the publication of this case report. WES of the subject and his unaffected parents was performed using the Agilent SureSelect Kit and Illumina HiSeq 2000 (PerkinElmer), covering $37.8 \times$ for the subject, $30.4 \times$ for the mother, and $27.5 \times$ for the father. The sequencing reads were mapped to the hg19 human reference genome. Rare variants, identified as those with minor allele frequency $(<0.01)$, were assessed for predicted impact on protein function and screened under multiple inheritance models, as described previously (31). Sanger sequencing was performed to confirm segregation with disease.

Analysis of free Neu5Ac levels. Levels of urinary sialic acids were determined by tandem mass spectrometry as reported previously $(32,33)$. Fibroblast pellets ( 2.5 million cells, obtained after trypsinization) in $250 \mu \mathrm{l}$ of $50 \mathrm{mM}$ Tris/ $\mathrm{HCl}$ ( $\mathrm{pH} 7.5$ ) were sonicated on ice ( 3 times 8 seconds), and then centrifuged for 10 minutes at $10,000 \mathrm{~g}$ and $4^{\circ} \mathrm{C}$. RBCs, washed in $0.9 \% \mathrm{NaCl}$, were sonicated on ice ( 3 times 8 seconds), and then centrifuged for 10 minutes at $10,000 \mathrm{~g}$ and $4^{\circ} \mathrm{C}$. To $100 \mu 1$ of the supernatant, ${ }^{13} \mathrm{C} 3-N$-acetyl-neuraminic acid (50 $\mu$, Merck) was added as internal standard and the solution was filtered using a $30-\mathrm{kDa}$ filter 


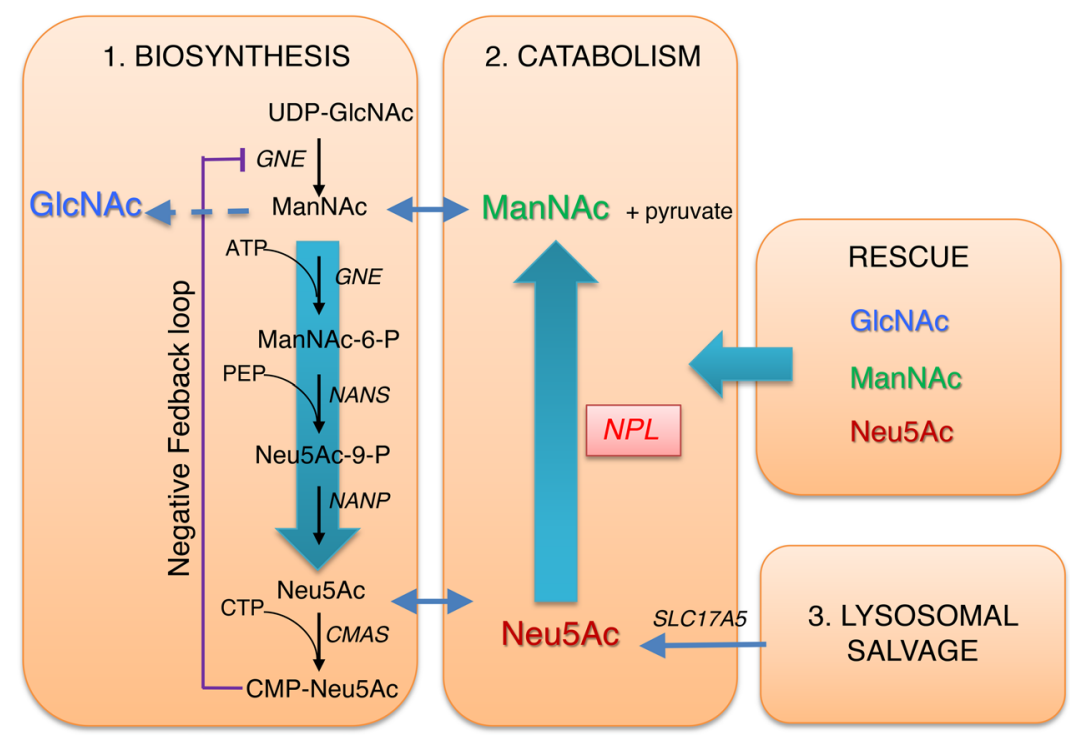

Figure 10. Roles of NPL in sialic acid catabolism and potential monosaccharide replacement therapy. Neu5Ac homeostasis is known to involve a biosynthetic pathway (1) starting from UDP-GIcNAc and a salvage pathway (2) in which Neu5Ac released from glycoproteins and glycolipids in the lysosome can be used for CMP-Neu5Ac synthesis. Our data lead us to hypothesize that Neu5Ac catabolism also has physiological relevance in this homeostasis and its downstream metabolites are important for muscle function.

(Amicon Ultra) into tubes containing $10 \mu 1$ of $2 \%$ formic acid. After centrifugation (30 minutes for fibroblasts, 40 minutes for RBCs) at $13,000 \mathrm{~g}$ and $4^{\circ} \mathrm{C}$, the flow-through was used for quantification of Neu5Ac by mass spectrometry as described previously $(1,2)$. Assays were performed in duplicate and Neu5Ac levels were normalized for protein level in the lysates.

NPL activity in human cells. The lysates of cultured fibroblasts and RBCs obtained as described above were used for protein determination and enzyme activity assay. Ten microliters of a $5 \mathrm{mM}$ solution of Neu5Ac (Carbosynth), $50 \mu \mathrm{l}$ of cell lysate, and $30 \mu 1$ of $50 \mathrm{mM}$ Tris/HCl buffer ( $\mathrm{pH} 7.5$ ) were mixed and incubated at $37^{\circ} \mathrm{C}$. The reactions were stopped by freezing at $-20^{\circ} \mathrm{C}$. The samples $(100 \mu \mathrm{l})$ were then supplemented with $50 \mu 1$ of ${ }^{13} \mathrm{C} 3-\mathrm{N}$-acetyl-neuraminic acid (Merck) as internal standard and the Neu5Ac levels were quantified by mass spectrometry as described above.

Analysis of NPL metabolites in patient cells. Analysis of NPL metabolites was performed based on previously described methods with minor modification (17). Briefly, to extract metabolites from RBCs, 35 $\mu 1$ of cell homogenate was incubated with $1,400 \mu 1$ extraction buffer $(2: 2: 1$ acetonitrile/methanol/water $[\mathrm{v} / \mathrm{v} / \mathrm{v}]$ ) for 5 minutes at $-20^{\circ} \mathrm{C}$. Fibroblasts were seeded in 6-well plates and upon reaching $70 \%$ confluence were washed twice with $75 \mathrm{mM}$ ammonium carbonate buffer ( $\mathrm{pH} 7.4$ ), followed by snap freezing in liquid nitrogen. To extract metabolites, wells were incubated twice with $700 \mu 1$ extraction buffer for 2 and 3 minutes, respectively, at $-20^{\circ} \mathrm{C}$. All samples were centrifuged at 16,000 $\mathrm{g}$ for 3 minutes followed by drying of the supernatant in a vacuum centrifuge at room temperature. Samples were dissolved in $50 \mu \mathrm{l}$ (RBCs) or $100 \mu \mathrm{l}$ (fibroblasts) LC/MS-grade water. Samples were analyzed using reverse-phase ion pairing chromatography (Agilent Technologies 1290 Infinity) coupled to a triple-quadrupole mass spectrometer operating in negative ion mode (Agilent Technologies 6490 Triple Quad LC/MS). Metabolites were separated on an Acquity UPLC column (Waters, HSS T3 $1.8 \mu \mathrm{m}, 2.1 \times 150 \mathrm{~mm}$ ) using a gradient of solvent A (10 mM tributylamine in water) and solvent B (10 mM tributylamine acid in methanol). Flow rate was $0.25 \mathrm{ml} / \mathrm{min}$ and injection volume was $2 \mu 1$. Temperatures of the autosampler and column compartment were kept stable at $7^{\circ} \mathrm{C}$ and $35^{\circ} \mathrm{C}$, respectively. Metabolites were analyzed using the following MRM transitions: HexNAc $(220.1 \rightarrow 119.0 \mathrm{~m} / z)$, ManNAc-6P $(300.1 \rightarrow 78.9 \mathrm{~m} / z)$, and Neu5Ac $(308.1 \rightarrow 87.0 \mathrm{~m} / z)$.

Expression and functional analysis of NPL mutant variants in HEK293T cells. Plasmids expressing NPL mutant variants were constructed using a QuikChange Lightning kit (Stratagene) with pcDNA3.1+/NPL-C-(K)DYK plasmid (OHu08401, GenScript) expressing human DYK-tagged NPL as a matrix. The mutagenesis primers were designed using the QuikChange Primer Design Program (http://www.stratagene.com/qcprimerdesign). 
HEK293T cells (ATCC) were cultured in DMEM supplemented with 10\% (v/v) fetal calf serum (Wisent). Transfections were carried out using Lipofectamine LTX (Invitrogen) as described in the manufacturer's protocol. Forty-eight hours after transfection, cells were washed with ice-cold PBS, and homogenized in $\mathrm{H}_{2} \mathrm{O}$ by sonication. To study NPL protein stability, 48 hours after transfection cells were washed with PBS and incubated for 2, 4, 6, 18, and 24 hours in DMEM with $10 \%$ (v/v) fetal calf serum and $7 \mu \mathrm{M}$ cycloheximide to inhibit de novo protein synthesis. After incubation, cells were washed in PBS, harvested, and stored at $-20^{\circ} \mathrm{C}$ until all time points were collected. Cell pellets were resuspended in $200 \mu \mathrm{l}$ of RIPA buffer ( $50 \mathrm{mM}$ Tris $\mathrm{HCl}, 150 \mathrm{mM}$ $\mathrm{NaCl}, 1 \%$ Nonidet P-40, 0.25\% Na-deoxycholate, 0.1\% SDS, 2 mM EDTA, 1 mM PMSF), supplemented with protease and phosphatase inhibitor cocktail (Roche), sonicated for 2 times 10 seconds and cleared by centrifugation at 13,000 $\mathrm{g}$ for 10 minutes. The protein was quantified using the Bradford reagent (Bio-Rad).

NPL enzymatic activity in cultured cells or fish embryos was measured against Neu5Ac (Sigma-A1drich), Neu5Gc (Sigma-Aldrich), or KDN (provided by A.J. Bennet, Simon Fraser University, Burnaby, B.C., Canada) as described by Brunetti et al. (15). The concentration of the product, ManNAc, was determined as described by Reissig et al. (34). $N$-acetyltransferase enzymatic activity was measured using the fluorogenic substrate 4-methylumbelliferyl $\beta$-D-glucosaminide (4MU- $\beta \mathrm{GlcN}$, Moscerdam), as previously described by $\mathrm{He}$ et al. (35).

For Western blot after separation by SDS-PAGE on an $8 \%$ gel, the proteins were transferred to a nitrocellulose membrane and hybridized with monoclonal rat antibodies against the DYK tag (637301, BioLegend, 1:5,000), rabbit polyclonal anti-HGSNAT antibodies (HPA029578, Sigma-Aldrich, 1:1,000), or monoclonal mouse anti- $\beta$-actin antibodies (47778, Santa Cruz Biotechnology, 1:1,000) followed by HPR-conjugated secondary antibodies (7077, 1:8,000 or 7074, 1:10,000; both Cell Signaling Technology). The membrane was developed with Pierce ECL Western Blotting substrate (Thermo Fisher Scientific) and the signal detected using the G:Box Chemi XQR system (Syngene). The intensities of bands were quantified using ImageJ software (NIH).

Zebrafish husbandry. Zebrafish embryos of the WT and cmlc2:GFP lines were maintained at $28.5^{\circ} \mathrm{C}$ with a cycle of 10 hours/14 hours.

Zebrafish morpholino gene knockdown. To knock down $n p l$ gene expression, we used $n p l$-atg MO and $n p l$-sp $\mathrm{MO}$ and a standard control MO (Cont MO). The MO sequences are as follows: $n p l$-atg MO 5'-CGCGTTGAGACATTTCTTCCTTTCA-3', npl-sp MO 5'-ATGTGCTTTAACTCACTTGCCCTTT-3', and Cont MO 5'-CCTCTTACCTCAGTTACAATTTATA-3'.

MOs were designed by Gene Tools, LLC. Both MOs were injected individually and in combination into 1-cell-stage zebrafish embryos. $n p l$-atg $(8 \mathrm{ng} / \mu \mathrm{l})$ and $n p l$-sp $(8 \mathrm{ng} / \mu \mathrm{l}) \mathrm{MOs}$ were injected individually and each injection was repeated at least 3 times.

Knockdown confirmation by RT-PCR. Knockdown of $n p l$ by $n p l$-sp was confirmed by RT-PCR. Embryos were injected at the 1 -cell stage with $8 \mathrm{ng} / \mu \mathrm{l}$ of each MO diluted in water (RNAse, DNAse free) containing phenol red (Sigma-Aldrich). Two hours after the injection, the embryos were sorted and those that incorporated phenol red in the animal pole were selected. At $24 \mathrm{hpf}$, embryos were manually dechorionated. Total RNA was extracted from embryos at $48 \mathrm{hpf}$ using TRIzol (Invitrogen). The RNA concentration of each sample was quantified using a NanoDrop ND-1000 spectrophotometer (NanoDrop Technologies). RNA integrity and band expected were verified using 1\% agarose gel electrophoresis. The RNA template was converted into cDNA using Superscript II reverse transcriptase (Invitrogen). The primers used are as follows: $n p l$ forward 5'-CAGCTACATTCACTCCACTCAC-3' and $n p l$ reverse 3'-CATGCCGAGCCAGTTCTT-5'.

In situ hybridization. RNA in situ hybridization in zebrafish embryos was performed as described previously (36). Zebrafish $n p l$ antisense and sense probes were generated by PCR using template cDNA obtained from RNA of zebrafish embryos at $48 \mathrm{hpf}$. PCR was used to produce copies of the transcript ENSDART00000011258 (npl). This PCR product was used to amplify a second product containing the T3 and T7 promoters in the $5^{\prime}$ and $3^{\prime}$ end, respectively. The riboprobe generated corresponds to $351 \mathrm{bp}$ of ensemble transcript ID. $n p l$ transcripts were PCR amplified targeting the transcript ENSDART00000011258. The primers used for amplification and the primers amplifying the $\mathrm{T} 3$ and $\mathrm{T} 7$ promoter containing products are as follows: $n p l$ forward 5'-CGAGAGGGAGATTCCATCATTT-3', $n p l$ reverse 3'-CCCATTGGTAACCCTGATACTT-5', $n p l$ T7 forward 5'-TAATACGACTCACTATAGGGAAAAGAGAAACGAGAGGGAGATTCCATCATTT-3', and npl T3 reverse 3'-ATTAACCCTCACTAAAGGAAAAGGAGGCCCATTGGTAACC CTGATACTT-5'. 
RNA in situ hybridization in zebrafish embryos was performed as described previously (37). All in situ hybridization experiments were repeated at least 3 times for each probe and stage.

Western blotting. Larvae injected with Cont $\mathrm{MO}$ and $n p l$-atg $\mathrm{MO}$ at 3 days after injection (20 larvae/ group) were homogenized in $50 \mathrm{mM}$ Tris buffer ( $\mathrm{pH}$ 7.5) containing protease inhibitor (Protease Inhibitor Cocktail Tablets, Roche). Protein concentration was measured using the bicinchoninic acid (BCA) method with bovine serum albumin (BSA) as the standard. Total protein (40 $\mu \mathrm{g}$ ) was separated in $10 \%$ SDS-PAGE, transferred to nitrocellulose membranes, and hybridized with anti-NPL rabbit polyclonal antibody (Abcam, catalog ab139057, 1:250 dilution) followed by HRP-labeled anti-rabbit secondary antibody (Santa Cruz Biotechnology; 1:3,000 dilution). Protein bands were identified with ECL detection and imaged using Image Lab detection system (Bio-Rad). Protein band intensity was quantified using ImageJ software. Equal loading was confirmed by incubation of membranes with Cy3-conjugated monoclonal mouse $\beta$-actin antibody (Sigma-Aldrich, catalog C5838, 1:1,000) for 1 hour at room temperature.

$M O$ injection and rescue experiments. Human NPL mRNA was obtained using the mMessage mMachine T7 kit (Ambion) from the clone U7323BE160-1 in the pcDNA3.1+/C-(K)DYK vector (GenScript). The same vector was used to create human NPL mutations pAsn45Asp and pArg63Cys and mutant NPL mRNA was synthesized using the same protocol (Ambion). Zebrafish embryos at the 1-cell stage were coinjected with $4 \mathrm{ng}$ of $n p l$-atg MO and $50 \mathrm{pg}$ of NPL mRNA WT and $4 \mathrm{ng}$ of $n p l$-atg MO and $50 \mathrm{pg}$ of human NPL mRNA with double mutation in 63C and 45D (MUT 63C + 45D), or single mutations (NPL mRNA MUT 63C), (NPL mRNA MUT 45D).

Zebrafish embryo immunofluorescence. Whole-mount immunofluorescence in zebrafish was performed as described below, zebrafish embryos at $48 \mathrm{hpf}$ were fixed with $4 \%$ paraformaldehyde (PFA) overnight at $4^{\circ} \mathrm{C}$. After fixation, 5 washes were conducted in PBS. All reactions were performed at room temperature (with gentle shaking) by sequential incubations in the following order: $10 \%$ normal goat serum (NGS), $1 \%$ BSA in PBS for 2 hours; washed 3 times 5 minutes each in PBST; then fluorescent (Alexa Fluor 488) phalloidin, diluted 1:1,000, for 2 hours. After three 15-minute washes in PBS, zebrafish embryos were mounted between 2 coverslips separated by petroleum jelly grease, in fresh mounting medium.

Zebrafish larva locomotion behavior assay. Zebrafish larvae at $2 \mathrm{dpf}$ were placed in individual wells of a 12-well plate in embryo medium (E2) and placed in a Zebrabox (ViewPoint Life Sciences) for video recording. The larvae were lightly touched with the tip of an Eppendorf Microloader and the behavior was recorded using the equipped camera at a frame rate of $30 \mathrm{~Hz}$. The locomotor behavior was then analyzed using the manual tracking plugin of ImageJ, and the total distance swam, maximum velocity, and swim duration of each larva were calculated. Each zebrafish larva was touched 10 times in order to determine its escape response behavior. For each condition, a sample size of 6-9 larvae was used to determine the mean and standard deviation of the behavior responses.

Zebrafish assay for oxidative stress. ROS levels in zebrafish larvae were visualized using the ROS indicator 2',7'-dichlorodihydrofluorescein diacetate (DCFH-DA; Sigma-Aldrich), as previously described (37). Briefly, embryos were injected at the 1-cell stage with Cont MO, npl-atg MO, or npl-atg MO plus WT human $N P L$ mRNA. Some of the embryos injected with the npl-atg MO were then incubated with ManNAc (800 $\mu \mathrm{M}) 2$ hours after injection. At $2 \mathrm{dpf}$, all the embryos were incubated with $100 \mu \mathrm{M}$ DCFH-DA for 1 hour at $28.5^{\circ} \mathrm{C}$ in the dark, and washed 3 times for 5 minutes each with embryo water. Images were acquired using a Leica DFC 300-FX camera and a Leica fluorescence microscope, and were processed with Fiji analysis software. Corrected total fluorescence $(\mathrm{CTF})$ was calculated using the following formula: $\mathrm{CTF}=$ integrated density - (area of selected cell $\times$ mean fluorescence of background readings). For each experimental group, $n=10-11$ fish per treatment. The experiment was repeated 2 times.

Microscopy. Live embryos were imaged in bright field and epifluorescence mode using fluorescent dissection microscopy (Leica M205 FA); fixed samples were imaged using confocal microscopy with a Zeiss laser-scanning microscope (LSM 700). Live embryos were anesthetized using $0.16 \mathrm{mg} / \mathrm{ml}$ tricaine methanesulfonate (Sigma-Aldrich) and embedded in 2.5\% methylcellulose (Sigma-Aldrich). Embryos fixed with $4 \%$ PFA were embedded in 1\% low-melting-temperature agarose (BioShop). Images were analyzed with LSM software (Zeiss), LAS FA software (Leica), Photoshop CS3 (Adobe), and ImageJ.

Statistics. Student's $t$ tests and 1-way ANOVA with appropriate post hoc tests were performed using GraphPad Prism software version 7 for statistical analyses and detailed test information is indicated in the figure legends. A linear mixed-effects model was used for the zebrafish larva locomotor behavior assay, with $n p l$ MO injection conditions (fixed effect) and individual larvae (random effect) with 
Bonferroni's post hoc test, using the R statistical package (https://www.r-project.org/). $P<0.05$ was considered statistically significant $\left({ }^{*} P<0.05, * * P<0.01,{ }^{* *} P<0.001\right)$.

Study approval. All zebrafish studies were approved by the Animal Care Committee of St. Michael's Hospital under the protocol number ACC660. TIDEX gene discovery study was approved by the University of British Columbia Research Ethics Board (IRB H12-00067).

\section{Author contributions}

XYW, MTG, AVP, CDMVK, and DJL designed the research. MTG, KBA, AW, BR, KH, XP, ADS, SER, RN, KS, AMP, JY, FZ, AAB, WWW, MH, FK, UE, ADB, and RAW performed the experiments and/ or analysis of the data. XCY, CJR, AML, BD, JM, HV, MVS, MH, and CDMVK contributed to clinical studies. DJL, XYW, AVP MTG, BR, and CDMVK wrote the manuscript.

\section{Acknowledgments}

We gratefully acknowledge the patient and his family for participation in this study; our colleagues at BC Children's Hospital and Vancouver General Hospital for clinical management of the patient; X. Han for Sanger sequencing; B. Sayson and Aisha Ghani for consenting and data management; Dora Pak and Evelyn Lomba for administration and coordination; and M. Higginson for DNA extraction, sample handling, and technical data (University of British Columbia). The authors acknowledge funding support from Natural Sciences and Engineering Research Council of Canada, grant RGPIN 05389-14 (to XYW), Brain Canada Foundation and Health Canada, grant PSG14-3505 (to XYW), and Canada Foundation for Innovation, grant 26233 (to XYW). This work was also supported by funding from the BC Children's Hospital Foundation as "1st Collaborative Area of Innovation" (www.tidebc.org), the Genome BC (SOF-195 grant), the Canadian Institutes of Health Research (grants 301221 and PJT-148863) and Rare Diseases Network Foundation (27R21814 to AVP). Informatics infrastructure was supported by Genome $\mathrm{BC}$ and Genome Canada (ABC4DE Project). CDMVK is a recipient of the Michael Smith Foundation for Health Research Scholar Award. This work was also financially supported by grants from the Dutch Organization for Scientific Research, ZONMW (Medium Investment Grant 40-00506-98-9001 and VIDI Grant 91713359 to DJL) and by the European Union's Horizon 2020 research and innovation program (EUROCDG-2) under the ERA-NET Cofund action number 643578. The authors acknowledge data analysis support from biostatistician Gerald Lebovic from the Applied Health Research Centre (AHRC) of St. Michael's Hospital. G. Lebovic is also an Assistant Professor at the Institute of Health Policy, Management and Evaluation at the University of Toronto.

Address correspondence to: Xiao-Yan Wen, Zebrafish Centre for Advanced Drug Discovery, St. Michael's Hospital, 209 Victoria Street, Toronto, Ontario, Canada M5B 1T8. Phone: 416.847.1737; Email: x.wen@ utoronto.ca. Or to: Alexey V. Pshezhetsky, Sainte-Justine University Hospital Research Center, 3175 Cote Ste-Catherine, Montréal, Quebec, Canada, H3T 1C5. Phone: 514.345.4931 ext 2736; Email: alexei.pchejetski@umontreal.ca. Or to: Clara DM van Karnebeek, Amsterdam UMC, Department of Pediatrics, Emma Children's Hospital (Room H7-224), Academic Medical Center, Meibergdreef 9, 1105 AZ Amsterdam, Netherlands. Phone: 31.205662113; Email: c.d.vankarnebeek@amc.uva.nl. Or to: Dirk J. Lefeber, Radboud University Medical Center - 830, Geert Grooteplein 10, 6525 GA Nijmegen, Netherlands. Phone: 31.24.3093487; Email: Dirk.Lefeber@radboudumc.nl.

1. Varki A. Sialic acids in human health and disease. Trends Mol Med. 2008;14(8):351-360.

2. Kean EL, Münster-Kühnel AK, Gerardy-Schahn R. CMP-sialic acid synthetase of the nucleus. Biochim Biophys Acta. 2004;1673(1-2):56-65.

3. Eckhardt M, Mühlenhoff M, Bethe A, Gerardy-Schahn R. Expression cloning of the Golgi CMP-sialic acid transporter. Proc Natl Acad Sci USA. 1996;93(15):7572-7576.

4. Harduin-Lepers A, Vallejo-Ruiz V, Krzewinski-Recchi MA, Samyn-Petit B, Julien S, Delannoy P. The human sialyltransferase family. Biochimie. 2001;83(8):727-737.

5. Monti E, et al. Sialidases in vertebrates: a family of enzymes tailored for several cell functions. Adv Carbohydr Chem Biochem. 2010;64:403-479.

6. Pan X, et al. Neuraminidases 3 and 4 regulate neuronal function by catabolizing brain gangliosides. FASEB J. 2017;31(8):3467-3483

7. Schauer R, Sommer U, Krüger D, van Unen H, Traving C. The terminal enzymes of sialic acid metabolism: acylneuraminate pyruvate-lyases. Biosci Rep. 1999;19(5):373-383.

8. Willems AP, van Engelen BG, Lefeber DJ. Genetic defects in the hexosamine and sialic acid biosynthesis pathway. Biochim Biophys 
Acta. 2016;1860(8):1640-1654.

9. van Karnebeek CDM, et al. Corrigendum: NANS-mediated synthesis of sialic acid is required for brain and skeletal development. Nat Genet. 2017;49(6):969.

10. Shyr C, Tarailo-Graovac M, Gottlieb M, Lee JJ, van Karnebeek C, Wasserman WW. FLAGS, frequently mutated genes in public exomes. BMC Med Genomics. 2014;7:64.

11. Kumar P, Henikoff S, Ng PC. Predicting the effects of coding non-synonymous variants on protein function using the SIFT algorithm. Nat Protoc. 2009;4(7):1073-1081.

12. Adzhubei I, Jordan DM, Sunyaev SR. Predicting functional effect of human missense mutations using PolyPhen-2. Curr Protoc Hum Genet. 2013; Chapter 7:Unit7.20.

13. Kircher M, Witten DM, Jain P, O'Roak BJ, Cooper GM, Shendure J. A general framework for estimating the relative pathogenicity of human genetic variants. Nat Genet. 2014;46(3):310-315.

14. Hrebícek M, et al. Mutations in TMEM76* cause mucopolysaccharidosis IIIC (Sanfilippo C syndrome). Am J Hum Genet. 2006;79(5):807-819.

15. Brunetti P, Jourdian GW, Roseman S. The sialic acids. III. Distribution and properties of animal N-acetylneuraminic aldolase. J Biol Chem. 1962;237:2447-2453.

16. Coene KLM, et al. Next-generation metabolic screening: targeted and untargeted metabolomics for the diagnosis of inborn errors of metabolism in individual patients. J Inherit Metab Dis. 2018;41(3):337-353.

17. Buescher JM, Moco S, Sauer U, Zamboni N. Ultrahigh performance liquid chromatography-tandem mass spectrometry method for fast and robust quantification of anionic and aromatic metabolites. Anal Chem. 2010;82(11):4403-4412.

18. Delgado-Olguín P, et al. CTCF promotes muscle differentiation by modulating the activity of myogenic regulatory factors. $J$ Biol Chem. 2011;286(14):12483-12494.

19. Cho A, et al. Sialic acid deficiency is associated with oxidative stress leading to muscle atrophy and weakness in GNE myopathy. Hum Mol Genet. 2017;26(16):3081-3093.

20. Wang DY, et al. Endoplasmic reticulum resident protein 44 (ERp44) deficiency in mice and zebrafish leads to cardiac developmental and functional defects. J Am Heart Assoc. 2014;3(5):e001018.

21. Shi X, et al. Effect of empagliflozin on cardiac biomarkers in a zebrafish model of heart failure: clues to the EMPA-REG OUTCOME trial? Mol Cell Biochem. 2017;433(1-2):97-102.

22. Pace CN, Scholtz JM. A helix propensity scale based on experimental studies of peptides and proteins. Biophys J. 1998;75(1):422-427.

23. Nees S, Schauer R, Mayer F. Purification and characterization of N-acetylneuraminate lyase from Clostridium perfringens Hoppe-Seyler's Z Physiol Chem. 1976;357(6):839-853.

24. Izard T, Lawrence MC, Malby RL, Lilley GG, Colman PM. The three-dimensional structure of N-acetylneuraminate lyase from Escherichia coli. Structure. 1994;2(5):361-369.

25. Bergfeld AK, Pearce OM, Diaz SL, Pham T, Varki A. Metabolism of vertebrate amino sugars with N-glycolyl groups: elucidating the intracellular fate of the non-human sialic acid N-glycolylneuraminic acid. J Biol Chem. 2012;287(34):28865-28881.

26. Lines MA, et al. Infantile sialic acid storage disease: Two unrelated inuit cases homozygous for a common novel SLC17A5 mutation. JIMD Rep. 2014;12:79-84.

27. Adams D, Gahl WA. Free sialic acid storage disorders. In: Pagon RA, et al. eds. GeneReviews. Seattle (WA): University of Washington, Seattle; 2003. https://www.ncbi.nlm.nih.gov/books/NBK1470/.

28. Niethamer TK, et al. Oral monosaccharide therapies to reverse renal and muscle hyposialylation in a mouse model of GNE myopathy. Mol Genet Metab. 2012;107(4):748-755.

29. Asaka T, et al. Homozygosity and linkage disequilibrium mapping of autosomal recessive distal myopathy (Nonaka distal myopathy). J Hum Genet. 2001;46(11):649-655

30. Moulin M, Ferreiro A. Muscle redox disturbances and oxidative stress as pathomechanisms and therapeutic targets in early-onset myopathies. Semin Cell Dev Biol. 2017;64:213-223.

31. Tarailo-Graovac M, et al. Exome sequencing and the management of neurometabolic disorders. N Engl J Med. 2016;374(23):2246-2255.

32. Valianpour F, Abeling NG, Duran M, Huijmans JG, Kulik W. Quantification of free sialic acid in urine by HPLC-electrospray tandem mass spectrometry: a tool for the diagnosis of sialic acid storage disease. Clin Chem. 2004;50(2):403-409.

33. van der Ham M, de Koning TJ, Lefeber D, Fleer A, Prinsen BH, de Sain-van der Velden MG. Liquid chromatography-tandem mass spectrometry assay for the quantification of free and total sialic acid in human cerebrospinal fluid. J Chromatogr B Analyt Technol Biomed Life Sci. 2010;878(15-16):1098-1102.

34. Reissig JL, Storminger JL, LeLoir LF. A modified colorimetric method for the estimation of N-acetylamino sugars. J Biol Chem. 1955;217(2):959-966.

35. He W, et al. Prenatal diagnosis of Sanfilippo disease type C using a simple fluorometric enzyme assay. Prenat Diagn. 1994;14(1):17-22.

36. Stainier DY, Gilbert W. Pioneer neurons in the mouse trigeminal sensory system. Proc Natl Acad Sci USA. 1990;87(3):923-927.

37. Philip AM, et al. Development of a zebrafish sepsis model for high-throughput drug discovery. Mol Med. 2017;23:134-148. 